RUSSIAN JOURNAL OF EARTH SCIENCES, VOL. 4, NO. 2, PAGES 103-119, APRIL 2002

\title{
Siberian craton - a fragment of a Paleoproterozoic supercontinent
}

\author{
O. M. Rosen \\ Geological Institute (GIN), Russian Academy of Sciences
}

\begin{abstract}
The sialic (volcanogenic) material of the terranes in 3the Siberian fragment of a Paleoproterozoic supercontinent separated from the mantle 3.5, 3.3, 3.0, and 2.5 billion years ago. The collision zones (sutures) between the terranes are dated by granitoids which melted from the crust of the terranes in two periods of time: 1.9 and 1.8 billion years ago. Local metamorphism and granite generation inside the collision (fault) zones were accompanied by synchronous areal dry granulite metamorphism in the adjacent terranes. This implies a fairly uniform field of high temperatures and pressures typical of the thicker crust of the collision prism. The amalgamation of the continental-crust fragments was terminated by the formation of a huge mountain massif ca. 1.8 billion years ago. Later, these mountains were wholly eroded. Early Riphean platform-type clastic sediments started to accumulate on the resulting peneplain 1.65 billion years ago, i. e., 150 million years after the end of the collision. The Siberian Craton is a part of the Pangea-1 Paleoproterozoic supercontinent which originated from the fragments of the broken Archean Pangea-0 supercontinent which had existed as microcontinents with their own sedimentary-volcanic covers which were transformed, after the collision, to the foldbelts superimposed over the basements of the old terranes. The exception was the Paleoproterozoic Akitkan volcanic belt which evolved first as a volcanic arc and later, in the course of the total amalgamation, was thrust over the ancient basement and involved in anorogenic acid magmatism (A-granites).
\end{abstract}

\section{Introduction}

The newly formed supercontinents were huge collisiontype mountains resulting from the amalgamation of various blocks of the sialic crust. The older were the supercontinents, the less evidence of their existence remained in the geologic records. The Paleoproterozoic supercontinent concerned, obviously the second, in terms of its origin, in the Earth's history, preserved enough evidence of its existence. However, no reliable map of its paleostructure has yet been offered. The results of this study open up new possibilities for this reconstruction. The mountains that arose during the collision have been long since eroded, and the presentday erosion surface shows merely the rocks of the intermediate and low crust. These are high-grade metamorphic rocks which were the subject of our reconstruction. This paper is

Copyright 2002 by the Russian Journal of Earth Sciences.

Paper number TJE02090.

ISSN: $1681-1208$ (online)

The online version of this paper was published 26 April 2002. URL: http://rjes.wdcb.ru/v04/TJE02090/TJE02090.htm based on the interpretation of geological, geochemical, and isotope-geochronological data available for the metamorphic rocks and collision granites, this making the results somewhat conventional. However, the good knowledge of the craton allows one to classify the conclusions derived as a fairly definite and consistent system of historico-geological phenomena.

The Siberian Craton occupies an area of roughly $4 \cdot 10^{9} \mathrm{~km}^{2}$ and is covered $(70 \%)$ by Phanerozoic sedimentary rocks ranging between 1 and $8 \mathrm{~km}$ and averaging about $4 \mathrm{~km}$ in thickness. In the north and east, the craton is surrounded by the essentially sedimentary foldbelts of the Phanerozoic foreland (Taimyr and Verkhoyansk belts), and in the west and south, by volcanic orogenic belts: the Paleozoic CentralAsia and Mesozoic Mongolia-Okhotsk belts.

\section{Terranes and Collision Zones as the Main Units of the Historico-Geological Analysis}

Most of the tectonic maps of the Siberain Craton were based on the analysis of mainly geological and/or geophysi- 
cal data (for example, a summary in [Yanshin and Borukaev, 1988; and others]). Later, the use of isotope data resulted in deriving historico-geological models for the craton's evolution [Dobretsov, 1986; Frost et al., 1998; Popov and Smelov, 1996; Rosen et al., 1994, 2000; Rundkvist and Mitrofanov, 1988; Smelov et al., 1998; and others]. A view was advanced that the terranes of different types and ages had been the main structural elements of the craton, which had originated and evolved initially, independently of one another, as isolated sialic masses (microcontinents) and later, as a result of a collision, had been amalgamated to one craton.

The faults separating the terranes show indications of tectonic compression and overthrusting and are classified as the zones of collision (sutures). Another necessary indication of collision boundaries between the terranes is the synchronism of local metamorphism and granitization in collision zones (sutures) and areal metamorphism in the adjacent terranes. This synchronism might have originated only as the result of thermal relaxation (spontaneous heating) inside the thickened crust of the collision prism, as follows from theoretical modeling [England and Thompson, 1984] and geological observations [Rosen and Fedorovskii, 2001].

The present-day structure of the craton is generally interpreted as the result of the collision and amalgamation (accretion) of microcontinents of different ages, which were transformed to different-type tectonic blocks (terranes) [Rosen et al., 1994, 2000; Smelov et al., 1998]. This process coincided in age with the amalgamation of other cratons and indicates that a supercontinent existed at that time.

The basement of the craton consists of two major structural elements, namely, of granulite-gneiss and granitegreenstone associations [Petrov et al., 1985], which, as indicated by recent data, have different ages and compose the terranes of the respective compositions. Differences between them are especially obvious, when different tectonotypes are compared in terms of variations in their rock associations and also in the specific patterns of magnetic and gravity anomalies they produce [Rosen et al., 2000]. It is obvious that the granulite-gneiss associations (primarily granulites) are lower-crust rocks, whereas the granite-greenstone formations are upser-crust complexes, dominated by the amebiform areas of granitoids (80-90\%) with the synforms composed of basic volcanics and graywackes. Although both rock types can be juxtaposed in the erosion surface of one terrane, the adopted binary separation of terranes seems to be a necessary simplification and a useful instrument of study until new geological and geochronological data are obtained.

\section{Structure of the Siberian Craton Basement}

The structural elements of the first order are tectonic provinces (superterranes). The essence of these two synonyms is the fact that prior to being included into the structure of the craton the terranes were grouped into large geologic bodies, and the collision and amalgamation of the latter resulted in the subsequent formation of the craton. The Tungusska, Anabar, Olenek, Aldan, and Stanovoi provinces have been identified. They consist of different-type terranes (tectonic blocks) and include Early Proterozoic orogenic foldbelts. A specific position is occupied by the Akitkan volcanic belt separating the Anabar and Aldan provinces.

The distribution of the tectonic provinces in the limits of the Siberian Craton is shown in Figure 1. The terranes and foldbelts are described here after [Rosen et al., 1994] and more recent publications. Table 1 includes almost all of the known dates of the Siberian Craton basement $(>220)$ and is the first attempt of such synthesis, known in the recent years. Preference is given to the $\mathrm{U}-\mathrm{Pb}$ and $\mathrm{Sm}-\mathrm{Nd}$ methods. The results obtained by other methods (K-Ar, Rb-Sr) are cited only in the cases of sufficiently numerous measurements using a uniform object (K-Ar) or in the cases of data that are in good agreement with more reliable dates ( $\mathrm{Rb}-\mathrm{Sr})$. The obvious discrepancies between the dates of large homogeneous objects were largely due to the contamination by the older (or younger) material of the crust $\left(\mathrm{T}_{N d}(\mathrm{DM})\right.$ model age determined by the Sm-Nd method), or by the heterogeneity of zircon crystals (U-Pb method with a concordia). Here, we discuss the most representative dates, showing a good agreement among them, the authors of which provided all necessary and fairly well determined geological and petrologic characteristics. The more numerous dates, which are fairly correct in terms of isotopic analysis, but are devoid of any distinct geological and petrological analysis of the authors, are used for comparison.

The largest Tungus Province is situated in the western half of the carton, where the larger area is occupied by the Tungus granite-greenstone terrane. The terrane is covered by the platform sediments and outcrops only in the south, within the Sharyzhalgai Uplift. Here, the volcanics and sedimentary rocks of the Onot greenstone belt are intruded by tonalites (3.25 Ga old), which were slightly metamorphosed 2.7-2.8 Ga ago [Rosen et al., 1994]. The belt is surrounded by granulites with a metamorphic age of $2.4-2.5$ Ga [Aftalion et al., 1991]. The whole rock complex was intruded by collision granites 1.95-1.96 and 1.82-1.87 Ga ago (a review in [Mekhonoshin, 1999]). During these periods of time the deeply metamorphosed mafic-ultramafic rocks, representing the fragments of the subducted oceanic crust [Sklyarov et al., 1998] and, possibly, of the lower crust and lithospheric mantle [Sharkov et al., 1995], were moved along the faults into the collision zone.

The Angara Foldbelt [Nozhkin, 1999; Rosen et al., 1993] borders the Tungus Terrane on the west and southwest and is exposed in the Yenisey Range and in the Sayan region. It is traceable under the sediments northward as far as the Taimyr Peninsula thanks to its negative linear magnetic anomalies and low gravity values. Its granulite basement originated $2.73 \mathrm{Ga}$ ago and contains charnockite mobilizates with an age of $1.84 \mathrm{Ga}$. Its metavolcanics (biotite-hornblende gneiss) originated there simultaneously with granite-gneiss domes 1.9-2.2 Ga ago [Nozhkin, 1999], and the Sayan and Tarak huge linear granite massifs were intruded along the southern and western boundaries of the Tungus Terrane approximately $1.78-1.90$ Ga ago. The association of the rocks of the Archean basement, the Early Proterozoic volcanic rocks, and the huge banded granite plutons surrounding the craton suggests that the conditions of 


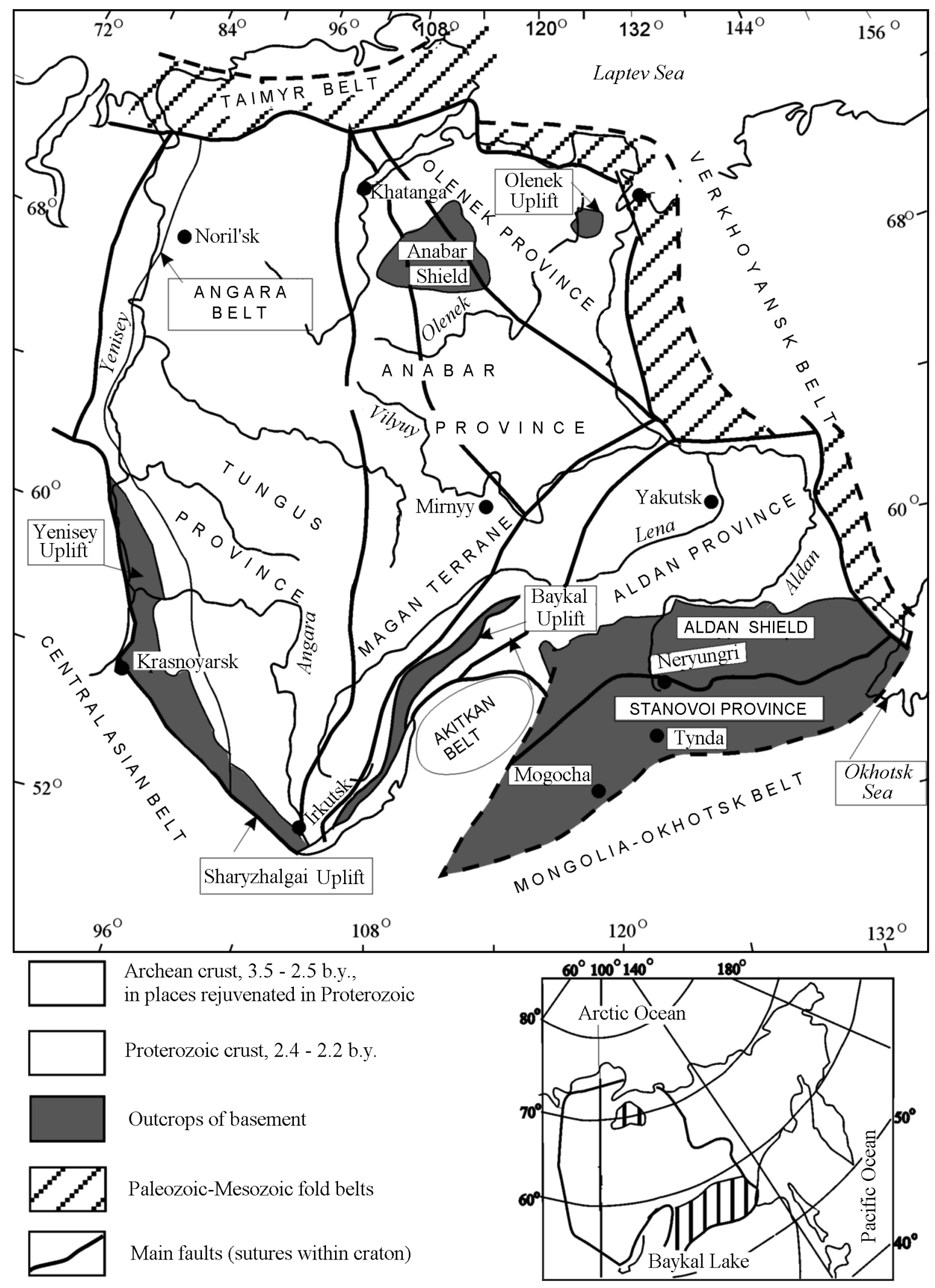

Figure 1. Principal structural elements of the Siberian Craton basement (modified after [Rosen et al., 1994]), see the text for explanations. 
Table 1. Geochronological data for the Early Precambrian of the Siberian Craton

\begin{tabular}{|c|c|c|c|c|}
\hline Date, Ma & Method & Material & Rock, geological position & Reference \\
\hline \multicolumn{5}{|c|}{ Tungusska Province } \\
\hline \multicolumn{5}{|c|}{ Tungusska Terrane (Sharyzhalgai fragment) } \\
\hline 3250 & $\mathrm{U}-\mathrm{Pb}$ & zircon, u.i. ${ }^{*}$ & tonalite, Onot Belt & [Rosen et al., 1994] \\
\hline 2776 & $\mathrm{U}-\mathrm{Pb}$ & zircon, u.i.* & xenocryst in charnockite, Sharyzhalgai Complex & [Rosen et al., 1994] \\
\hline 2784 & $\mathrm{U}-\mathrm{Pb}$ & zircon, u.i. ${ }^{*}$ & charnockite, Sharyzhalgai Complex & [Rosen et al., 1994] \\
\hline 2775 & & & same & [Rosen et al., 1994] \\
\hline 2710 & & & same & [Rosen et al., 1994] \\
\hline 2700 & & & same & [Rosen et al., 1994] \\
\hline 2700 & & & same & [Rosen et al., 1994] \\
\hline 2568 & $\mathrm{U}-\mathrm{Pb}$ & zircon, u.i.* & $\begin{array}{l}\text { kinzigite, metamorphic zircon, } \\
\text { Sharyzhalgai Complex }\end{array}$ & [Rosen et al., 1994] \\
\hline 2560 & $\mathrm{U}-\mathrm{Pb}$ & zircon, u.i.* & same & [Rosen et al., 1994] \\
\hline 2540 & $\mathrm{Rb}-\mathrm{Sr}$ & bulk sample & charnockite, Sharyzhalgai Complex & [Rosen et al., 1994] \\
\hline 2530 & $\mathrm{Rb}-\mathrm{Sr}$ & bulk sample & same & [Rosen et al., 1994] \\
\hline 2400 & $\mathrm{~T}_{\mathrm{Nd}}(\mathrm{DM})$ & bulk sample & charnockite, Sharyzhalgai Complex & [Rosen et al., 1994] \\
\hline 2300 & & & same & [Rosen et al., 1994] \\
\hline 1965 & $\mathrm{U}-\mathrm{Pb}$ & zircon, u.i. ${ }^{*}$ & granite, Sharyzhalgai Complex & [Rosen et al., 1994] \\
\hline 1950 & $\mathrm{U}-\mathrm{Pb}$ & zircon, u.i. ${ }^{*}$ & same & [Rosen et al., 1994] \\
\hline 1873 & & & same & [Rosen et al., 1994] \\
\hline 1870 & & & same & [Rosen et al., 1994] \\
\hline 1870 & & & same & [Rosen et al., 1994] \\
\hline 1862 & $\mathrm{U}-\mathrm{Pb}$ & monazite & same, concordant value & [Rosen et al., 1994] \\
\hline 1817 & $\mathrm{U}-\mathrm{Pb}$ & zircon, u.i.* & same & [Rosen et al., 1994] \\
\hline \multicolumn{5}{|c|}{ Angara Belt } \\
\hline 2730 & $\mathrm{U}-\mathrm{Pb}$ & zircon, u.i. ${ }^{*}$ & orthopyroxene gneiss, Yenisey tectonic window & [Rosen et al., 1994] \\
\hline 1900 & $\mathrm{U}-\mathrm{Pb}$ & zircon, u.i.* & granulite, same site & [Rosen et al., 1994] \\
\hline 1840 & $\mathrm{U}-\mathrm{Pb}$ & zircon, u.i.* & charnockite, same site & [Rosen et al., 1994] \\
\hline 2750 & $\mathrm{~Pb}-\mathrm{Pb}$ & zircon & metadacite, Yukseevo greenstone belt & [Rosen et al., 1994] \\
\hline 2760 & $\mathrm{~Pb}-\mathrm{Pb}$ & zircon & gneiss, Biryusa tectonic window & [Rosen et al., 1994] \\
\hline 1926 & $\mathrm{U}-\mathrm{Pb}$ & zircon & charnockite, same site, concordant value & [Rosen et al., 1994] \\
\hline 1760 & $\mathrm{~Pb}-\mathrm{Pb}$ & zircon & pegmatite, same site & [Rosen et al., 1994] \\
\hline 1750 & & & same & [Rosen et al., 1994] \\
\hline 1750 & & & same & [Rosen et al., 1994] \\
\hline 1740 & & & same & [Rosen et al., 1994] \\
\hline 1880 & $\mathrm{U}-\mathrm{Pb}$ & zircon, u.i.* & biotite-hornblende gneiss, Yenisey sequence & [Rosen et al., 1994] \\
\hline 1780 & $\mathrm{U}-\mathrm{Pb}$ & zircon, u.i.* & same, at contact with Tarak granite & [Rosen et al., 1994] \\
\hline 1760 & $\mathrm{U}-\mathrm{Pb}$ & zircon, u.i. ${ }^{*}$ & rapakivi granite, Gareevo Complex & [Rosen et al., 1994] \\
\hline 1900 & $\mathrm{U}-\mathrm{Pb}$ & zircon, u.i. ${ }^{*}$ & granite, Sayany Complex & [Rosen et al., 1994] \\
\hline \multicolumn{5}{|c|}{ Anabar Province } \\
\hline \multicolumn{5}{|c|}{ Magan Terrane } \\
\hline 3010 & $\mathrm{~T}_{N d}(\mathrm{DM})$ & bulk sample & $\begin{array}{l}\text { orthopyroxene gneiss and metavolcanics, } \\
\text { Vyurbyur Group }\end{array}$ & [Rosen et al., 2000] \\
\hline 3090 & $\mathrm{~T}_{N d}(\mathrm{DM})$ & bulk sample & metagraywacke, calciphyre, same site & [Rosen et al., 2000] \\
\hline 3000 & & & same & [Rosen et al., 2000] \\
\hline 2940 & & & same & [Rosen et al., 2000] \\
\hline 2900 & & & same & [Rosen et al., 2000] \\
\hline 2850 & & & same & [Rosen et al., 2000] \\
\hline 2840 & & & same & [Rosen et al., 2000] \\
\hline 2800 & & & same & [Rosen et al., 2000] \\
\hline 3010 & $\mathrm{~T}_{\mathrm{Nd}}(\mathrm{DM})$ & bulk sample & $\begin{array}{l}\text { amphibolite, xenolith in kimberlite of Mir pipe, } \\
\text { Mir kimberlite field }\end{array}$ & [Rosen et al., 2000] \\
\hline 2420 & $\mathrm{U}-\mathrm{Pb}$ & zircon, u.i. ${ }^{*}$ & two pyroxene-plagioclase gneiss (metavolcanite) & [Rosen et al., 2000] \\
\hline
\end{tabular}


Table 1. Continued

\begin{tabular}{|c|c|c|c|c|}
\hline \multicolumn{5}{|c|}{ Kotuikan fault (collision) zone } \\
\hline 1920 & $\mathrm{U}-\mathrm{Pb}$ & monazite, u.i.* & syntectonic migmatite, Kotuikan R. area & [Rosen et al., 2000] \\
\hline 1950 & $\mathrm{~Pb}-\mathrm{Pb}$ & uraninite & same & [Rosen et al., 2000] \\
\hline 1870 & $\mathrm{U}-\mathrm{Pb}$ & zircon, u.i. ${ }^{*}$ & biotite granite vein, same site & [Rosen et al., 2000] \\
\hline 1840 & $\mathrm{U}-\mathrm{Pb}$ & zircon, u.i. ${ }^{*}$ & microcline granite vein, B. Kuonamka R. area & [Rosen et al., 2000] \\
\hline \multicolumn{5}{|c|}{ Daldyn Terrane } \\
\hline 3100 & $\mathrm{Sm}-\mathrm{Nd}$ & $\begin{array}{l}\text { isochron from } \\
\text { bulk samples }\end{array}$ & $\begin{array}{l}\text { plagiogneiss and metabasite, } \\
\text { Daldyn Series, Kotuikan R. area }\end{array}$ & [Rosen et al., 2000] \\
\hline 3170 & $\mathrm{~T}_{\mathrm{Nd}}(\mathrm{DM})$ & bulk sample & $\begin{array}{l}\text { plagiogneiss, enderbite, and metabasite, } \\
\text { Daldyn Group Nalim-Rassokha R. }\end{array}$ & [Rosen et al., 2000] \\
\hline 3160 & $\mathrm{~T}_{\mathrm{Nd}}(\mathrm{DM})$ & bulk sample & $\begin{array}{l}\text { quartzite, metagraywacke, and calciphyre, } \\
\text { same series, same site }\end{array}$ & [Rosen et al., 2000] \\
\hline 3130 & & & same & [Rosen et al., 2000] \\
\hline 3000 & & & same & [Rosen et al., 2000] \\
\hline 2280 & & & same & [Rosen et al., 2000] \\
\hline 3270 & $\mathrm{~T}_{\mathrm{Nd}}(\mathrm{DM})$ & bulk sample & $\begin{array}{l}\text { amphibolite and plagiogneiss of crustal } \\
\text { xenoliths in kimberlites } \\
\text { of Muna field }\end{array}$ & [Rosen et al., 2000] \\
\hline 3120 & & & same & [Rosen et al., 2000] \\
\hline 2920 & & & same & [Rosen et al., 2000] \\
\hline 2900 & & & same & [Rosen et al., 2000] \\
\hline 3350 & $\mathrm{U}-\mathrm{Pb}$ & zircon, u.i.* & metabasite, Daldyn Group, B. Kuonamka R. & [Rosen et al., 2000] \\
\hline 3320 & SHRIMP & zircon & same & [Rosen et al., 2000] \\
\hline 3000 & $\mathrm{U}-\mathrm{Pb}$ & zircon, u.i. ${ }^{*}$ & $\begin{array}{l}\text { plagiogneiss-metandesite, Daldyn Group, } \\
\text { B. Kuonamka R. }\end{array}$ & [Rosen et al., 2000] \\
\hline 2760 & $\mathrm{U}-\mathrm{Pb}$ & zircon, u.i.* & Daldyn-group plagiogneiss, metamorphic zircon & [Rosen et al., 2000] \\
\hline 1940 & $\mathrm{Sm}-\mathrm{Nd}$ & miner. isochron & garnet-plagioclase gneiss, Kotuikan R. head & [Rosen et al., 2000] \\
\hline 1900 & $\mathrm{Sm}-\mathrm{Nd}$ & miner. isochron & enderbite, Nalim-Rassokha R. head & [Rosen et al., 2000] \\
\hline 1880 & $\mathrm{Sm}-\mathrm{Nd}$ & miner. isochron & $\begin{array}{l}\text { amphibolite, xenolith, Novinka pipe, } \\
\text { Muna kimberlite field }\end{array}$ & [Rosen et al., 2000] \\
\hline 1800 & $\mathrm{Sm}-\mathrm{Nd}$ & miner. isochron & mafic granulite, Daldyn Group, B. Kuonamka R. & [Rosen et al., 2000] \\
\hline \multicolumn{5}{|c|}{ Markha Terrane } \\
\hline 2520 & $\mathrm{~T}_{\mathrm{Nd}}(\mathrm{DM})$ & bulk sample & $\begin{array}{l}\text { garnet-two pyroxene gneiss from xenolith } \\
\text { in Udachnaya Pipe, Daldyn kimberlite field }\end{array}$ & [Rosen et al., 2000] \\
\hline 2480 & $\mathrm{~T}_{\mathrm{Nd}}(\mathrm{DM})$ & bulk sample & same & [Rosen et al., 2000] \\
\hline 1760 & $\mathrm{Sm}-\mathrm{Nd}$ & miner. isochron & same & [Rosen et al., 2000] \\
\hline \multicolumn{5}{|c|}{ Billyakh Fault (collision) zone } \\
\hline 1970 & $\mathrm{U}-\mathrm{Pb}$ & zircon, u.i. & $\begin{array}{l}\text { garnet gneiss (metagraywacke) of Khapchan Group } \\
\text { in the margin of the zone, metamorphic zircon }\end{array}$ & [Rosen et al., 2000] \\
\hline 1800 & $\mathrm{~K}-\mathrm{Ar}$ & mica, bulk samples & $\begin{array}{l}\text { subalkalic porphyry-like granodiorite from Billyakh Massif } \\
\text { between Nalim-Rassokha and B. Kuonamka rivers }\end{array}$ & [Rosen et al., 2000] \\
\hline \multicolumn{5}{|c|}{ Olenek Province } \\
\hline \multicolumn{5}{|c|}{ Birekta Terrane } \\
\hline 2320 & $\mathrm{~T}_{\mathrm{Nd}}(\mathrm{DM})$ & bulk sample & $\begin{array}{l}\text { garnet gneiss (metagraywacke), metacarbonate, } \\
\text { Khapchan belt }\end{array}$ & [Rosen et al., 2000] \\
\hline 2410 & & & same & [Rosen et al., 2000] \\
\hline 2410 & & & same & [Rosen et al., 2000] \\
\hline 2440 & & & same & [Rosen et al., 2000] \\
\hline 1920 & $\mathrm{Sm}-\mathrm{Nd}$ & miner. isochron & garnet gneiss (metagraywacke), same site & [Rosen et al., 2000] \\
\hline 1910 & $\mathrm{Sm}-\mathrm{Nd}$ & miner. isochron & garnet gneiss (metagraywacke), same site & [Rosen et al., 2000] \\
\hline 1980 & $\mathrm{~K}-\mathrm{Ar}$ & micas & greenschist acid volcanics, Aekite Foldbelt & [Rosen et al., 2000] \\
\hline 1850 & $\mathrm{~K}-\mathrm{Ar}$ & mica, bulk samples & granites, same site & [Rosen et al., 2000] \\
\hline
\end{tabular}


Table 1. Continued

\begin{tabular}{|c|c|c|c|c|}
\hline \multicolumn{5}{|c|}{ Akitkan volcanic belt } \\
\hline \multicolumn{5}{|c|}{ Volcanic rocks } \\
\hline $\begin{array}{l}2843 \\
2788 \\
2760 \\
2755 \\
2738 \\
2734 \\
2718 \\
2480 \\
1866 \\
2530 \\
2507 \\
2499 \\
1823 \\
2469 \\
2369 \\
1863 \\
1835 \\
1880\end{array}$ & $\begin{array}{l}\mathrm{T}_{\mathrm{Nd}}(\mathrm{DM}) \\
\mathrm{U}-\mathrm{Pb} \\
\mathrm{T}_{\mathrm{Nd}}(\mathrm{DM}) \\
\mathrm{T}_{\mathrm{Nd}}(\mathrm{DM}) \\
\mathrm{T}_{\mathrm{Nd}}(\mathrm{DM}) \\
\mathrm{U}_{-} \mathrm{Pb} \\
\mathrm{T}_{\mathrm{Nd}}(\mathrm{DM}) \\
\mathrm{T}_{\mathrm{Nd}}(\mathrm{DM}) \\
\mathrm{U}-\mathrm{Pb} \\
\mathrm{U}-\mathrm{Pb} \\
\mathrm{U}-\mathrm{Pb}\end{array}$ & $\begin{array}{l}\text { bulk sample } \\
\text { zircon, u.i.* } \\
\text { bulk sample } \\
\text { bulk sample } \\
\text { bulk sample } \\
\text { zircon, u.i.* } \\
\text { bulk sample } \\
\text { bulk sample } \\
\text { zircon, u.i.* } \\
\text { zircon, u.i.* } \\
\text { zircon, u.i. }{ }^{*}\end{array}$ & $\begin{array}{l}\text { rhyodacite, Malokosinskaya Suite } \\
\text { basaltic andesite, same site } \\
\text { andesite, same site } \\
\text { same } \\
\text { same } \\
\text { same } \\
\text { same } \\
\text { trachyrhyodacite, Domugda Fm. } \\
\text { volcanics, same site } \\
\text { trachyrhyodacite, Khibela Fm. } \\
\text { latite, Chai Fm. (Lambora Complex) } \\
\text { quartz latite, same site } \\
\text { same } \\
\text { metatrachyrhyolite, Ilovir Fm. } \\
\text { same } \\
\text { same } \\
\text { acid volcanics, Sarma Fm. } \\
\text { garnet-cordierite granulite, } \\
\text { W. Baikal shore }\end{array}$ & 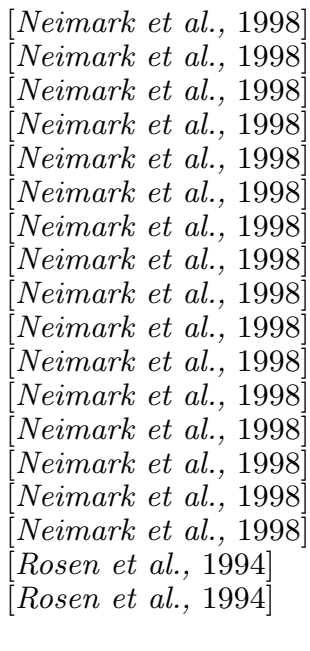 \\
\hline \multicolumn{5}{|c|}{ Granitoids } \\
\hline $\begin{array}{l}2369 \\
2326 \\
2073 \\
2073 \\
2066 \\
2020 \\
1998 \\
2490 \\
2490 \\
1866 \\
2353 \\
2446 \\
1871 \\
1864 \\
1910 \\
1910 \\
1890\end{array}$ & $\begin{array}{l}\mathrm{T}_{\mathrm{Nd}}(\mathrm{DM}) \\
\mathrm{T}_{\mathrm{Nd}}(\mathrm{DM}) \\
\mathrm{U}-\mathrm{Pb} \\
\mathrm{U}-\mathrm{Pb} \\
\mathrm{U}-\mathrm{Pb} \\
\mathrm{U}-\mathrm{Pb} \\
\mathrm{U}-\mathrm{Pb} \\
\mathrm{T}_{\mathrm{Nd}}(\mathrm{DM}) \\
\mathrm{T}_{\mathrm{Nd}}(\mathrm{DM}) \\
\mathrm{U}^{\mathrm{Pb}} \\
\mathrm{T}_{\mathrm{Nd}}(\mathrm{DM}) \\
\mathrm{T}_{\mathrm{Nd}}(\mathrm{DM}) \\
\mathrm{U}-\mathrm{Pb} \\
\mathrm{U}-\mathrm{Pb} \\
\mathrm{U}-\mathrm{Pb} \\
\mathrm{U}-\mathrm{Pb} \\
\mathrm{U}-\mathrm{Pb}\end{array}$ & $\begin{array}{l}\text { bulk sample } \\
\text { bulk sample } \\
\text { zircon, u.i.* } \\
\text { apatite, u.i.* } \\
\text { zircon, u.i.* } \\
\text { zircon, u.i.* } \\
\text { titanite, u.i.* } \\
\text { bulk sample } \\
\text { bulk sample } \\
\text { zirco, u.i.* } \\
\text { bulk sample } \\
\text { bulk sample } \\
\text { zircon, u.i.* } \\
\text { zircon, u.i.* } \\
\text { zircon, u.i.* } \\
\text { zircon, u.i.* } \\
\text { zircon, u.i.* }\end{array}$ & $\begin{array}{l}\text { granite gneiss, Chuya Complex } \\
\text { granite gneiss, same area } \\
\text { granite gneiss, same area } \\
\text { granite gneiss, same area } \\
\text { leucogranite, same area } \\
\text { granite gneiss, same area } \\
\text { granite gneiss, same area } \\
\text { quartz monzonite, Irel Complex } \\
\text { granite gneiss, same area } \\
\text { granite, same area } \\
\text { biotite granite, Abchad Complex } \\
\text { same } \\
\text { same } \\
\text { granite, Primorskii Complex } \\
\text { same } \\
\text { granite Kocherikovskii Complex } \\
\text { same }\end{array}$ & 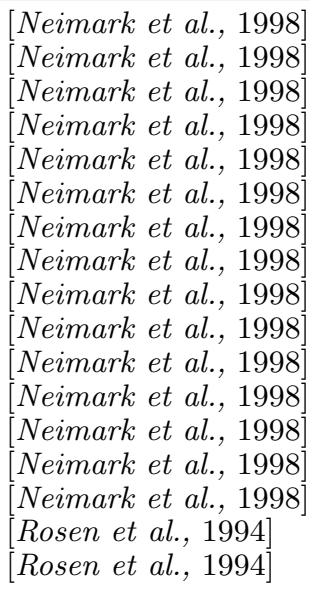 \\
\hline \multicolumn{5}{|c|}{ Aldan Province } \\
\hline \multicolumn{5}{|c|}{ Olekma Terrane } \\
\hline \multicolumn{5}{|c|}{ Tungurcha Greenstone Belt } \\
\hline $\begin{array}{l}3530 \\
3235 \\
3030 \\
3020 \\
2850 \\
3250 \\
3212 \\
3040 \\
3016 \\
2999 \\
2999 \\
2997 \\
2996 \\
2984 \\
2910 \\
2813\end{array}$ & $\begin{array}{l}\mathrm{T}_{\mathrm{Nd}}(\mathrm{DM}) \\
\mathrm{T}_{\mathrm{Nd}}(\mathrm{DM}) \\
\mathrm{T}_{\mathrm{Nd}}(\mathrm{DM}) \\
\text { Sm-Nd } \\
\text { SHRIMP } \\
\text { U-Pb } \\
\text { SHRIMP } \\
\text { SHRIMP } \\
\text { Pb-PB } \\
\text { U-Pb } \\
\text { SHRIMP } \\
\text { U-Pb } \\
\text { SHRIMP } \\
\text { Pb-Pb }\end{array}$ & $\begin{array}{l}\text { bulk sample } \\
\text { bulk sample } \\
\text { bulk sample } \\
\text { miner. isochron; } \\
\text { zircon } \\
\text { zircon, u.i.* } \\
\text { zircon } \\
\text { zircon } \\
\text { zircon } \\
\text { zircon, u.i.* } \\
\text { zircon } \\
\text { zircon, u.i.* } \\
\text { zircon } \\
\text { zircon }\end{array}$ & $\begin{array}{l}\text { tonalite gneiss } \\
\text { amphibolite } \\
\text { tonalite gneiss } \\
\text { same } \\
\text { tonalite } \\
\text { tonalite gneiss } \\
\text { gneiss } \\
\text { quartzite, Tasmiele sequence } \\
\text { tonalite gneiss, Khani R. } \\
\text { trondhjemite gneiss, Khani R. } \\
\text { syntectonic granite } \\
\text { quartzite, Tasmiele sequence } \\
\text { same } \\
\text { syntectonic granite } \\
\text { quartzite, Tasmiele sequence } \\
\text { tonalite gneiss, Khani R. }\end{array}$ & 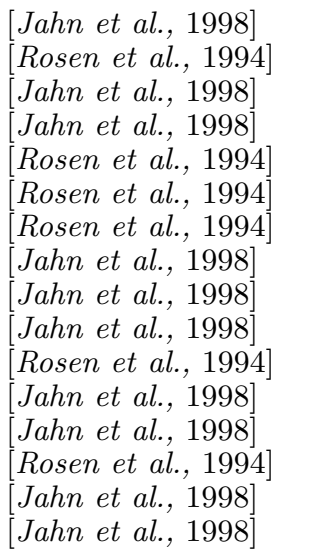 \\
\hline
\end{tabular}


Table 1. Continued

\begin{tabular}{|c|c|c|c|c|}
\hline \multicolumn{5}{|c|}{ Olondo Greenstone Belt } \\
\hline 3212 & SHRIMP & zircon & "ancient gneiss", Olekma R. & [Jahn et al., 1998] \\
\hline 3018 & SHRIMP & zircon & diorite gneiss & [Jahn et al., 1998] \\
\hline 3006 & SHRIMP & zircon & gneiss (metavolcanite) & [Jahn et al., 1998] \\
\hline 3006 & & & same & [Jahn et al., 1998] \\
\hline 2109 & $\mathrm{U}-\mathrm{Pb}$ & zircon, u.i.* & tonalite, Oldongso Massif & [Jahn et al., 1998] \\
\hline 2998 & SHRIMP & zircon & gneiss (metavolcanite) & [Jahn et al., 1998] \\
\hline 2984 & $\mathrm{U}-\mathrm{Pb}$ & zircon, u.i.* & tonalite, Amnunnatka Massif & [Jahn et al., 1998] \\
\hline 2983 & SHRIMP & zircon & gneiss (metavolcanite) & [Jahn et al., 1998] \\
\hline 2960 & $\mathrm{U}-\mathrm{Pb}$ & zircon, u.i.* & metavolcanite & [Jahn et al., 1998] \\
\hline 2862 & SHRIMP & zircon & tonalite gneiss & [Jahn et al., 1998] \\
\hline 2751 & SHRIMP & zircon & gneiss, Temulyakit Fault & [Rosen et al., 1994] \\
\hline 2738 & & & same & [Rosen et al., 1994] \\
\hline 3100 & $\mathrm{~T}_{\mathrm{Nd}}(\mathrm{DM})$ & bulk sample & trondhjemite gneiss & [Jahn et al., 1998] \\
\hline 3000 & $\mathrm{~T}_{\mathrm{Nd}}(\mathrm{DM})$ & bulk sample & diorite gneiss & [Jahn et al., 1998] \\
\hline 2370 & $\mathrm{~T}_{\mathrm{Nd}}(\mathrm{DM})$ & bulk sample & eclogite & [Jahn et al., 1998] \\
\hline 1947 & SHRIMP & zircon & tonalite gneiss & [Jahn et al., 1998] \\
\hline 1929 & & & same & [Jahn et al., 1998] \\
\hline 1895 & SHRIMP & zircon & gneiss, dated using overgrowths on zircons & [Rosen et al., 1994] \\
\hline 3018 & $\mathrm{~T}_{\mathrm{Nd}}(\mathrm{DM})$ & bulk sample & $\begin{array}{l}\text { representative averaged age dating } \\
\text { the onset of greenstone-belt volcanism }\end{array}$ & [Rosen et al., 1994] \\
\hline 3018 & SHRIMP & zircon & same & [Rosen et al., 1994] \\
\hline 3000 & $\mathrm{~T}_{\mathrm{Nd}}(\mathrm{DM})$ & bulk sample & $\begin{array}{l}\text { representative averaged age dating } \\
\text { the end of volcanic activity }\end{array}$ & {$[$ Rosen et al., 1994] } \\
\hline 3000 & SHRIMP & zircon & same & [Rosen et al., 1994] \\
\hline 2950 & $\mathrm{~T}_{\mathrm{Nd}}(\mathrm{DM})$ & bulk sample & $\begin{array}{l}\text { representative averaged age dating } \\
\text { onset of metamorphism }\end{array}$ & [Rosen et al., 1994] \\
\hline 2950 & $\mathrm{U}-\mathrm{Pb}$ & zircon & same & [Rosen et al., 1994] \\
\hline 2860 & $\mathrm{Sm}-\mathrm{Nd}$ & $\begin{array}{l}\text { bulk sample } \\
\text { miner. isochron }\end{array}$ & $\begin{array}{l}\text { representative averaged age dating } \\
\text { tonalite magmatism }\end{array}$ & [Rosen et al., 1994] \\
\hline 2860 & SHRIMP & zircon & same & [Rosen et al., 1994] \\
\hline \multicolumn{5}{|c|}{ Aldan Terrane } \\
\hline 3570 & $\mathrm{U}-\mathrm{Pb}$ & zircon, u.i.* & tonalite gneiss of Iengra sequence & [Rosen et al., 1994] \\
\hline 3500 & $\mathrm{~T}_{\mathrm{Nd}}(\mathrm{DM})$ & bulk sample & granulite metagraywacke, Central Dome & [Kovach et al., 1999] \\
\hline 3500 & & & same & [Kovach et al., 1999] \\
\hline 3550 & $\mathrm{~T}_{\mathrm{Nd}}(\mathrm{DM})$ & bulk sample & tonalite gneiss & {$[$ Jahn et al., 1998] } \\
\hline 2940 & $\mathrm{~T}_{\mathrm{Nd}}(\mathrm{DM})$ & bulk sample & mafic granulite & [Jahn et al., 1998] \\
\hline 3080 & $\mathrm{~T}_{\mathrm{Nd}}(\mathrm{DM})$ & bulk sample & diorite gneiss & [Jahn et al., 1998] \\
\hline 2950 & & & same & [Jahn et al., 1998] \\
\hline 3280 & $\mathrm{~T}_{\mathrm{Nd}}(\mathrm{DM})$ & bulk sample & granite & [Jahn et al., 1998] \\
\hline 3640 & $\mathrm{~T}_{\mathrm{Nd}}(\mathrm{DM})$ & bulk sample & tonalite gneiss & [Jahn et al., 1998] \\
\hline 3540 & $\mathrm{~T}_{\mathrm{Nd}}(\mathrm{DM})$ & bulk sample & amphibolite & [Jahn et al., 1998] \\
\hline 3570 & $\mathrm{U}-\mathrm{Pb}$ & zircon, u.i.* & tonalite gneiss, Aldan R. & [Jahn et al., 1998] \\
\hline 3390 & $\mathrm{U}-\mathrm{Pb}$ & zircon, u.i.* & same & [Jahn et al., 1998] \\
\hline 3386 & $\mathrm{U}-\mathrm{Pb}$ & zircon, u.i.* & trondhjemite, Central Dome & [Rosen et al., 1994] \\
\hline 3335 & SHRIMP & zircon & tonalite gneiss, Aldan R. & [Jahn et al., 1998] \\
\hline 3335 & SHRIMP & zircon & acid nebulite granulite & [Rosen et al., 1994] \\
\hline 3328 & SHRIMP & zircon & same, dark round zircon cores & [Rosen et al., 1994] \\
\hline 3168 & $\mathrm{~Pb}-\mathrm{Pb}$ & zircon & tonalite gneiss, Aldan R. & [Jahn et al., 1998] \\
\hline 3160 & $\mathrm{~Pb}-\mathrm{Pb}$ & zircon & tonalite gneiss, Aldan R. & [Jahn et al., 1998] \\
\hline 1947 & SHRIMP & zircon & same, light prismatic zircon overgrowth & [Rosen et al., 1994] \\
\hline 3250 & $\mathrm{~T}_{\mathrm{Nd}}(\mathrm{DM})$ & bulk sample & orthopyroxene granulite, Central Dome & [Frost et al., 1998] \\
\hline 3100 & $\mathrm{~T}_{\mathrm{Nd}}(\mathrm{DM})$ & bulk sample & granulite metagraywacke, same site & [Kovach et al., 1999] \\
\hline
\end{tabular}


Table 1. Continued

\begin{tabular}{|c|c|c|c|c|}
\hline 3000 & $\mathrm{~T}_{\mathrm{Nd}}(\mathrm{DM})$ & bulk sample & orthopyroxene diorite, Timpton R. & [Kovach, 1994] \\
\hline 3000 & $\mathrm{~T}_{\mathrm{Nd}}(\mathrm{DM})$ & bulk sample & same, same site & [Kotov et al., 1999] \\
\hline 2800 & $\mathrm{~T}_{\mathrm{Nd}}(\mathrm{DM})$ & bulk sample & orthopyroxene diorite, Timpton R. & {$[$ Kovach, 1994] } \\
\hline 2740 & $\mathrm{~T}_{\mathrm{Nd}}(\mathrm{DM})$ & bulk sample & orthopyroxene granulite, Central Dome & [Frost et al., 1998] \\
\hline 2600 & $\mathrm{~T}_{\mathrm{Nd}}(\mathrm{DM})$ & bulk sample & granulite metagraywacke, same site & [Kovach et al., 1999] \\
\hline 2400 & & & same & [Kovach et al., 1999] \\
\hline 2400 & & & same & [Kovach et al., 1999] \\
\hline 2210 & $\mathrm{~T}_{\mathrm{Nd}}(\mathrm{DM})$ & bulk sample & granulite metapelite, same site & [Frost et al., 1998] \\
\hline 2180 & & & same & [Frost et al., 1998] \\
\hline 1950 & $\mathrm{U}-\mathrm{Pb}$ & zircon & orthopyroxene diorite, Timpton.R & [Frost et al., 1998] \\
\hline 1929 & SHRIMP & zircon prism & silicic granulite & [Rosen et al., 1994] \\
\hline 2220 & $\mathrm{~T}_{\mathrm{Nd}}(\mathrm{DM})$ & bulk sample & gabbro, Ungra Block & [Frost et al., 1998] \\
\hline 2037 & $\mathrm{U}-\mathrm{Pb}$ & zircon, u.i. ${ }^{*}$ & leucogabbro, same site & [Rosen et al., 1994] \\
\hline 2410 & $\mathrm{~T}_{\mathrm{Nd}}(\mathrm{DM})$ & bulk sample & granulite metagraywacke, Idzhek allochthone & [Kovach et al., 1999] \\
\hline 1916 & $\mathrm{U}-\mathrm{Pb}$ & zircon, u.i.* & charnockite, Idzhek zone & [Rosen et al., 1994] \\
\hline 1620 & $\mathrm{U}-\mathrm{Pb}$ & zircon & same & [Frost et al., 1998] \\
\hline 1700 & $\mathrm{U}-\mathrm{Pb}$ & zircon & gneiss & [Larin et al., 1997] \\
\hline 1840 & $\mathrm{~K}-\mathrm{Ar}$ & phlogopite & skarn, Central Dome & [Rosen et al., 1994] \\
\hline \multicolumn{5}{|c|}{ Udokam Belt } \\
\hline 2180 & $\mathrm{U}-\mathrm{Pb}$ & zircon, u.i. ${ }^{*}$ & tuffaceous sandstone & [Rosen et al., 1994] \\
\hline 1950 & $\mathrm{Rb}-\mathrm{Sr}$ & $\begin{array}{l}\text { isochrone from } \\
\text { bulk samples }\end{array}$ & siltstone & [Rosen et al., 1994] \\
\hline 1800 & $\mathrm{Rb}-\mathrm{Sr}$ & $\begin{array}{l}\text { isochrone from } \\
\text { bulk samples }\end{array}$ & granite cutting Udokan strata & [Rosen et al., 1994] \\
\hline 1780 & $\mathrm{~Pb}-\mathrm{Pb}$ & zircon & granite, Kodar Massif & [Rosen et al., 1994] \\
\hline \multicolumn{5}{|c|}{ Ulkan Belt } \\
\hline 1727 & $\mathrm{U}-\mathrm{Pb}$ & zircon, u.i.* & granite & [Rosen et al., 1994] \\
\hline 1727 & $\mathrm{U}-\mathrm{Pb}$ & zircon, u.i. ${ }^{*}$ & rhyolite & [Rosen et al., 1994] \\
\hline 1703 & $\mathrm{U}-\mathrm{Pb}$ & zircon, u.i.* & granite & [Rosen et al., 1994] \\
\hline \multicolumn{5}{|c|}{ Stanovoi Province } \\
\hline \multicolumn{5}{|c|}{ Sutam Terrane } \\
\hline 3460 & $\mathrm{U}-\mathrm{Pb}$ & zircon, u.i.* & zircon xenocrysts in metagabbro & [Rosen et al., 1994] \\
\hline 2200 & $\mathrm{U}-\mathrm{Pb}$ & zircon, l.i. ${ }^{*}$ & same sample & [Rosen et al., 1994] \\
\hline 3200 & $\mathrm{U}-\mathrm{Pb}$ & zircon, u.i.* & orthopyroxene plagiogneiss & [Rezvanova et al., 1994] \\
\hline 1980 & $\mathrm{U}-\mathrm{Pb}$ & zircon, l.i. ${ }^{*}$ & same sample & [Rezvanova et al., 1994] \\
\hline 3100 & $\mathrm{U}-\mathrm{Pb}$ & zircon, u.i.* & orthopyroxene plagiogneiss & [Shemyakin et al., 1998] \\
\hline 1940 & $\mathrm{U}-\mathrm{Pb}$ & zircon, l.i. ${ }^{*}$ & same sample & [Shemyakin et al., 1998] \\
\hline 2660 & $\mathrm{U}-\mathrm{Pb}$ & zircon, u.i.* & charnockite at contact with Kalar anorthosite & [Rosen et al., 1994] \\
\hline 1800 & $\mathrm{U}-\mathrm{Pb}$ & zircon, l.i. ${ }^{*}$ & same sample & [Rosen et al., 1994] \\
\hline 2900 & $\mathrm{Rb}-\mathrm{Sr}$ & $\begin{array}{l}\text { isochrone from } \\
\text { bulk samples }\end{array}$ & orthopyroxene plagiogneiss & [Mishkin et al., 1999] \\
\hline 3000 & $\mathrm{~T}_{\mathrm{Nd}}(\mathrm{DM})$ & bulk sample & orthopyroxene plagiogneiss & [Kotov et al., 1999] \\
\hline 2150 & $\mathrm{Sm}-\mathrm{Nd}$ & miner. isochron & plagiogneiss, Zverev Complex & [Rosen et al., 1994] \\
\hline 1950 & & & same & [Rosen et al., 1994] \\
\hline 3460 & $\mathrm{U}-\mathrm{Pb}$ & zircon, u.i.* & mafic granulite, Kurulta Complex & [Jahn et al., 1998] \\
\hline 3070 & $\mathrm{~T}_{\mathrm{Nd}}(\mathrm{DM})$ & bulk sample & tonalite gneiss, Kurulta Complex & [Jahn et al., 1998] \\
\hline \multicolumn{5}{|c|}{ Mogocha Terrane } \\
\hline 1873 & $\mathrm{U}-\mathrm{Pb}$ & zircon & amphibolite & [Rosen et al., 1994] \\
\hline 1950 & $\mathrm{U}-\mathrm{Pb}$ & zircon & paragneiss & [Rosen et al., 1994] \\
\hline 1930 & $\mathrm{U}-\mathrm{Pb}$ & zircon, u.i.* & migmatite granite & [Rosen et al., 1994] \\
\hline
\end{tabular}


Table 1. Continued

\begin{tabular}{|c|c|c|c|c|}
\hline \multicolumn{5}{|c|}{ Dzheltulak Belt } \\
\hline 2208 & $\mathrm{~K}-\mathrm{Ar}$ & & pegmatite & [Rosen et al., 1994] \\
\hline \multicolumn{5}{|c|}{ Tynda Terrane } \\
\hline 3220 & $\mathrm{~T}_{\mathrm{Nd}}(\mathrm{DM})$ & bulk sample & mafic granulite, Larba Complex & [Jahn et al., 1998] \\
\hline 3220 & & & mafic granulite, Larba Complex & {$[$ Jahn et al., 1998] } \\
\hline 2785 & SHRIMP & zircon & tonalite & [Rosen et al., 1994] \\
\hline 2785 & SHRIMP & zircon & tonalite gneiss, Gilyui R. & {$[$ Jahn et al., 1998] } \\
\hline 2585 & $\mathrm{U}-\mathrm{Pb}$ & zircon & kinzigite fragments, concordant values & [Rosen et al., 1994] \\
\hline 1960 & SHRIMP & zircon & tonalite, overgrowths on zircons & [Rosen et al., 1994] \\
\hline 1929 & SHRIMP & zircon & tonalite & [Rosen et al., 1994] \\
\hline 1929 & SHRIMP & zircon & metaharzburgite dike, Gilyui R. & {$[$ Jahn et al., 1998] } \\
\hline 1924 & SHRIMP & zircon & tonalite & [Rosen et al., 1994] \\
\hline 1924 & SHRIMP & zircon & mafic dike, Gilyui R. & [Jahn et al., 1998] \\
\hline 1734 & $\mathrm{U}-\mathrm{Pb}$ & zircon, u.i.* & anorthosite, Geran Massif & [Rosen et al., 1994] \\
\hline
\end{tabular}

Note: u.i. ${ }^{*}$ - upper discordia-concordia intersection; l.i. ${ }^{*}$ - lower discondia-concordia intersection.

an active continental margin existed there at the end of the early Proterozoic [Nozhkin, 1999; Rosen et al., 1993]. Apparently, these conditions continued to exist during the Riphean, when volcanic rocks $0.87 \mathrm{Ga}$ in age accumulated, and granite-gneiss domes were formed [Nozhkin, 1999]. Later, during the closure of the Riphean paleoocean in Vendian time, the Riphean-Vendian ophyolites and the island-arc volcanics of the Isakovka Complex [Vernikovskii, 1996] and the Predivino Terrane with an age of 0.64 Ga [Chernykh, 2000; Vernikovskii et al., 1999] were thrust over this Riphean active continental margin.

The Tungus Province is bounded in the east by the huge submeridional Sayan-Taimyr fault (collision) zone which divides the Siberian Craton into its eastern and western halves. This zone is traceable from under the platform sediments by an abrupt change in the mosaic geophysical fields, typical of the Tungus Terrane, to the linear patterns typical of the Magan Terrane. It is only in the south that this zone emerges to the surface west of Lake Baikal, where the late metamorphism of the rocks of the Sharyzhalgai Group is supposed to have been associated with the collision processes [Aftalion et al., 1991; Zonenshain et al., 1989].

The central and northeastern parts of the craton are occupied by the Anabar and Olenek provinces. The Anabar Province includes the Magan and Daldyn granulite-gneiss terranes, both outcropping in the Anabar Shield. The age of the rocks there is $3.1 \mathrm{Ga}$, the ages of the granulite metamorphism and granitoid melting being 1.8 and $1.9 \mathrm{Ga}$, respectively. The Anabar Province also includes the Markha granite-greenstone terrane covered by the platform sediments in the Markha $\mathrm{R}$. drainage area where granitoids were found in deep-hole cores. The accumulation of the rocks has been dated $2.5 \mathrm{Ga}$, and their metamorphism, 1.76 Ga (a review in [Rosen et al., 2000]).

The Kotuikan collision (fault) zone extends between the Daldyn and Magan terranes. Varying in width from a few kilometers to $30 \mathrm{~km}$, this zone is a collision-related blasto- mylonite macromelange. Its gneiss-migmatite apomylonite matrix (amphibolite metamorphic grade), containing concordant and cross-cutting vein-shaped autochthone granites, includes tectonic anorthosite blocks and large, measuring up to dozens of kilometers in length, granulite masses, which had been detached from the adjacent terranes and subject to regressive amphibolite metamorphism as a result of the hydrous fluids rising along the thrust surface (a review in [Luts and Oksman, 1990; Markov, 1988]). The characteristic elements of this tectonic melange are the band-shaped interlayers, concordant with the tectonite bedding, of sedimentogenic quartzite, carbonate, and apovolcanic high-Mg amphybolite, possibly representing the individual fragments of intercontinental basins, which had been involved into collision and acted as a low-viscosity substance (accretion wedge after [Dobretsov and Kirdyashkin, 1994]) at the front of the microcontinents' collision. The blastotectonites, as well as the migmatites and granitoids, generated during the collisionrelated thickening of the crust at the time of the terrane accretion, were dated 1.8 and $1.9 \mathrm{Ga}$, respectively [Rosen et al., 2000].

Some indirect evidence suggests the possibility of older collision events in this region. A boundary between the Markha Terrane (2.5 Ga old) and the Daldyn Terrane (3.1 Ga old), separating the crustal blocks of different ages, might have been a collision zone. Proceeding from the geological relationships presented in Figure 2, this zone must be substantially older than both collision zones bordering it in the east and west (Kotuikan and Billyakh zones, 1.8-2.0 Ga old). The collision of these terranes can be dated roughly as $2.3 \mathrm{Ga}$. Another date was derived from the interpretation of the relicts of the Archean granulite metamorphism (2.76 Ga [Rosen et al., 2000]) inside the Daldyn Terrane. Taking into consideration an association between the granulite metamorphism of the terranes and the processes of granite generation and metamorphism in the adjacent collision zones, it can be postulated that the value of $2.76 \mathrm{Ga}$ dates some earlier col- 


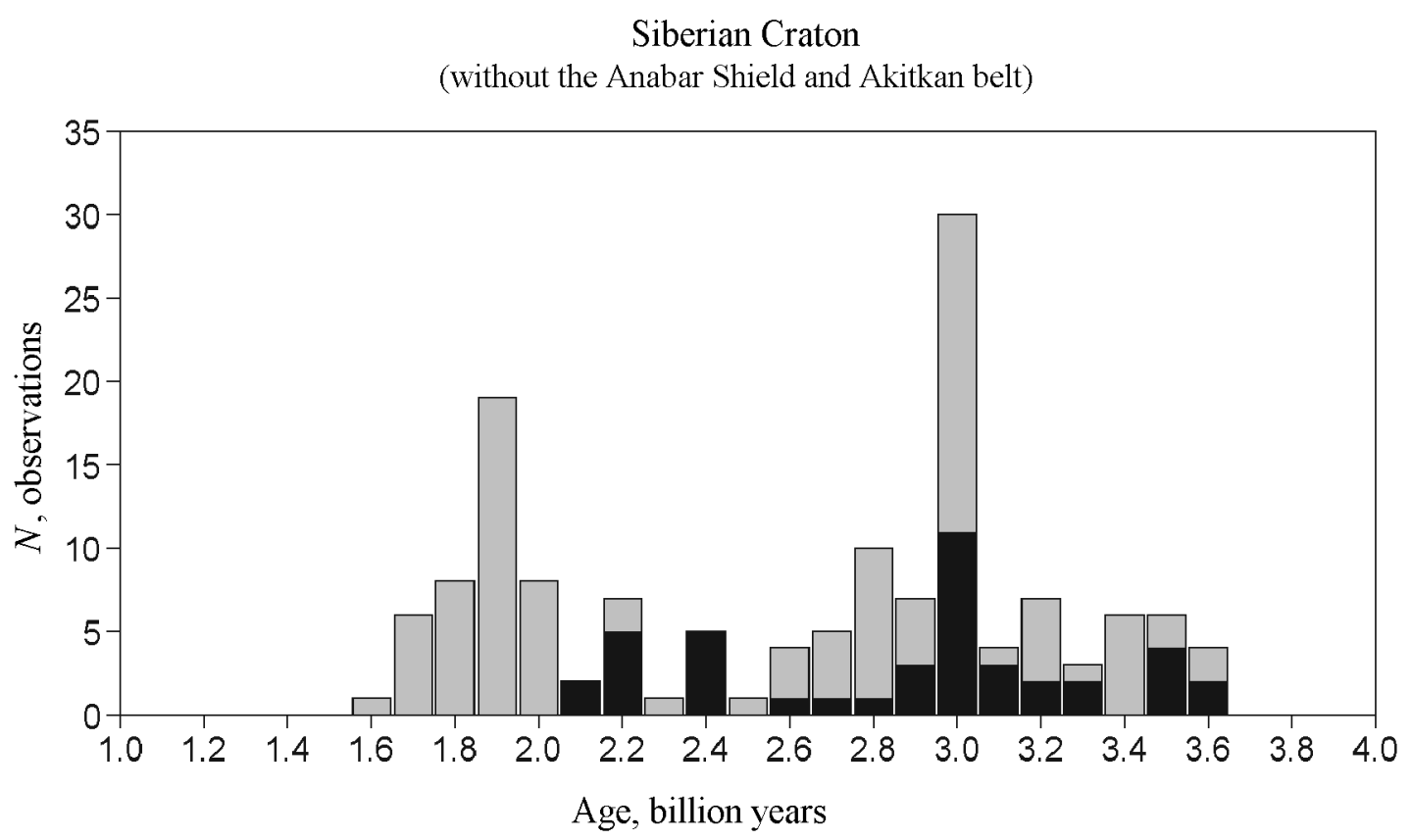

Sm-Nd model age, T(DM)Nd, $(N=42)$
U-Pb zircon age, $(N=102)$

Figure 2. Histogram of isotope-geochronological dates for the Siberian Craton (without the Anabar Shield and the Northeastern Territory), see the text for explanations.

lision event in the region, although the geometric configuration of the objects of this age is still unknown. Therefore, three successive collision epochs can be suggested for the Anabar Province: (1) a late epoch when granite generation in the collision zones and areal granulite metamorphism in the terranes occurred during two periods of time: $1.90-1.97$ and 1.76-1.87 Ga ago (Late Paleoproterozoic); (2) an intermediate epoch of roughly $2.3 \mathrm{Ga}$ ago (Early Paleoproterozoic); and (3) an earliest epoch, 2.8 Ga ago (Late Archeozoic).

The Billyakh collision (fault) zone separates the Anabar Province from the Olenek Province. The metamorphic rocks of the Khapchan Belt, associated with this collision zone, were dated $1.97 \mathrm{Ga}$, and the collision migmatites and granites were dated $1.8 \mathrm{Ga}$ [Rosen et al., 2000].

Olenek Province includes tectonic elements of various types and also a poorly known area of the so called Ust-Lena basement inlier in the northeastern margin of the craton. Outcropping in the west of the province, within the Anabar Shield, are the granulite metagraywackes (garnet gneiss) and metacarbonates (calcareous-silicate rocks and calciphyres) of the Khapchan Foldbelt. The source of the clastic material was dated $2.4 \mathrm{Ga}$, and the granulite metamorphism, $1.97 \mathrm{Ga}$ [Rosen et al., 2000]. It is obvious that the source of these rocks could not have been the rocks of the adjacent Daldyn Terrane dated $3.1 \mathrm{Ga}$ old. The object of erosion was obviously the basement of the Birekte granite-greenstone terrane (now wholly covered by the sediments), whereas the rocks of the Khapchan Belt accumulated on its passive (now western) margin. The rocks that accumulated on the Olenek Uplift, where the eastern (active) margin was located, were the acid volcanics and carbonaceous silicites of the Aekite Foldbelt, transformed to greenschist metamorphics $1.98 \mathrm{Ga}$ ago and intruded by granites roughly $1.85 \mathrm{Ga}$ ago.

Akitkan Volcanic Belt extends northeastward from the Cis-Baikalia region as far as the Lena River, where it is covered by the volcanogenic sediments of the Vilyui Paleozoic rift of the same NW strike. This belt cuts off, almost at a right angle, the SE-striking structures of the Anabar and Olenek provinces along the Vilyui fault zone, and is separated from the Aldan Province by the Lena fault zone. The oldest rocks there are the highly metamorphosed metavolcanics, quartzite, and carbonate (Chuya and Sarma suites), as well as the Chuya granites including zircons which have been dated 2.02-2.07 Ga, using the upper discordia intersection, with a $\mathrm{T}_{N d}(\mathrm{DM})$ 2.3-2.4 model age of the source, which had been recrystallized during a collision $1.91-2.00 \mathrm{Ga}$ ago [Neimark et al., 1998]. These indications suggest the belt to be an independent island-arc system of Late Paleoproterozoic age [Rosen et al., 1994]. Judging by the structural relationships (Figure 2), this fold zone must have been later thrust northwest over the rocks of the Anabar Province. Later, 1.83 and $1.87 \mathrm{Ga}$ ago, an anorogenic shoshonite-series potassic rock association was formed, known as the Akitkan volcanic and sedimentary rock group together with the Irel and Abchad comagmatic A-type granitoids [Neimark et al., 1998]. These Late Paleoproterozoic rocks accumulated in the 
environment of a marginal volcanoplutonic belt [Nozhkin, 1999], that is, as a volcanic arc on the active continental margin of the Anabar Superterrane. The later granitoids have been dated $0.32-0.73 \mathrm{Ga}$. The superimposed thermal events have been dated $0.45-0.5 \mathrm{Ga}$, which can be interpreted as the result of the effects of tectonic events in the Paleoasian Ocean and, in particular, in the vicinity of the Bargusin Terrane located east of the southern segment of the Akitkan Belt.

Situated in the southeast of the craton are the Aldan and Stanovoi provinces, separated by the Kalar (Stanovoi) thrust zone [Rosen et al., 1994]. Popov and Smelov [1996] and Smelov et al. [1998] analyzed the dates and petrology of their rocks. They believe that the Aldan (Aldan-Stanovoi) Shield was formed as the result of the successive accretion of the fragments of different orogenic belts and cratons to the Aldan and Tynda terranes over a period of 2.1 to $1.7 \mathrm{Ga}$.

Aldan Province includes the Olekma and Batomga granite-greenstone terranes, in the west and east, respectively, with the Aldan and Uchur granulite-gneiss terranes situated between them. The crust-formation processes in the Olekma granite-greenstone and Aldan granulite-gneiss terranes have been dated by the U-Pb method using zircons $\sim 3.0 \mathrm{Ga}$, the time of the subsequent transformations, $\sim 2.0 \mathrm{Ga}$, and the $\mathrm{T}_{N d}(\mathrm{DM})$ values obtained by the SmNd method were $3.6 \pm 0.1$ and $3.0 \pm 0.1$ Ga (a review in [Rosen et al., 1994]) and one value for the near-fault eclogite, 2.4 Ga [Smelov and Beryozkin, 1993]. When plotted in a ${ }^{207} \mathrm{~Pb} /{ }^{204} \mathrm{~Pb}-{ }^{206} \mathrm{~Pb} /{ }^{204} \mathrm{~Pb}$ diagram [Jahn and Cuvellier, 1998], the metacarbonates showed an isochrone of $2.02 \pm 0.08 \mathrm{Ga}$ [Jahn et al., 1998], this value obviously dating the last thermal event, namely a Proterozoic collision. The granulite plagiogneisses of the Aldan Terrane were dated later [Frost et al., 1998] as $\mathrm{T}_{N d}(\mathrm{DM}) 3.25 \mathrm{Ga}$ and $2.74 \mathrm{Ga}$, and the metapelites yielded a $\mathrm{T}_{N d}(\mathrm{DM})$ value (the integral age of the area of erosion) equal to $2.18-2.21 \mathrm{Ga}$ which turned out to be close to the intrusion time of basites in the Ungra block dated $\mathrm{T}_{N d}(\mathrm{DM}) 2.22 \mathrm{Ga}$. It appears that the Early Proterozoic igneous rocks had served as a source for some sedimentogenic granulite metamorphic rocks of the Aldan Terrane. The model datings of granulite metagraywackes from the Olekma, Aldan, and Uchur terranes [Kovach et al., 1999] yielded the average ages of the rocks from the erosion area to be $\mathrm{T}_{N d}(\mathrm{DM}) 3.5,3.0$, and $2.6 \mathrm{Ga}$, these values corresponding to the dates of the adjacent infracrustal rocks which might have served as a source for the dated clastic material. The dates of $2.2-$ $2.4 \mathrm{Ga}$ reported by these authors for the metaterrigenous rocks of the same terranes suggest a potential erosion of the Early Proterozoic rocks (not found yet) and call for a further more detailed interpretation. The dating of metagraywacks from an erosion area in the Idzhek allochthone yielded the T(DM)ND values of 2.1-2.4 Ga [Kovach et al., 1999], which do not contradict the $1.92-1.77 \mathrm{Ga}$ age of the metamorphism, yet suggest that the adjacent terranes could not have been the source of the clastic material. If this is the case, the Idzhek allochthone is a fragment of some still unknown independent Paleoproterozoic terrane. Resting on the granitegreenstone basement of the Olekma and Batomga terranes are the volcanogenic and sedimentary rocks of the platform cover, belonging to the Udokan (a review in [Rosen et al., 1994]) and Ulkan [Larin et al., 1997] foldbelts. The rocks of the former accumulated $2.2 \mathrm{Ga}$ ago, obviously, in a foredeep that had formed at the front of the Stanovoi Superterrane at the beginning of its movement to the north (in the presentday structure) along the Kalar thrust zone. It is possible, however, that the Udokan deposits had accumulated as a platform cover on the Olekma microcontinent as far back as prior to the accretion. The rocks of the other, Ulkan, complex seem to have filled, $1.7 \mathrm{Ga}$ ago, an intermontane trough inside the preexisting orogenic belt of the Aldan tectonic province.

A collision between the Aldan and Uchur terranes along the Timpton and Tyrkanda fault zones was dated $1.92 \mathrm{Ga}$ by the time of the Idzhek charnokite melting [Bibikova et al., 1986; Frost et al., 1998] and $1.95 \mathrm{Ga}$ by the time of orthopyroxene diorite melting in the Timpton R. drainage area (with the model ages of the source ranging between $\mathrm{T}_{N d}(\mathrm{DM}) 2.8$ and $\left.3.0 \mathrm{Ga}\right)$ [Kovach, 1994].

Stanovoi Province is situated on the southeastern margin of the Siberian Craton. In the southwest it includes the Mogocha granite-greenstone terrain (its granite formation was dated $1.93 \mathrm{Ga}$, its last metamorphism, $1.87 \mathrm{Ga}$ ) and in the east, the Tynda terrane which includes the domains of amphibolite metamorphic facies (Stanovoi Complex) and of granulite facies (Larba Complex) (a review in [Rosen et al., 1994]). The tonalite intrusion was dated $2.78 \mathrm{Ga}$ (from a source 3.22 Ga old), and the superimposed metamorphism, $1.96 \mathrm{Ga}$. Pressed between these two terranes 2.0 Ga ago was the Dzheltulak Foldbelt composed of clastic deposits, dolomite, and basalt interlayers, metamorphosed to amphibolite and greenschist facies. Both terranes are thrust northward, along the Stanovoi fault zone, over the Sutam Terrain which is thrust, in its turn, along the Kalar fault zone over the Aldan Province terranes, adjacent in the north.

The Sutam granulite-gneiss terrane is distinguished by the high content (up to 40\%) of basites (Zverev Complex [Godzevich, 1986]) and by its high-pressure granulite metamorphism (Kurulta Complex). A characteristic feature is the presence of basic-ultrabasic rock and anorthosite massifs (e.g., Kalar Massif) extending as far as $50 \mathrm{~km}$ in length. The zircon xenocrysts in the metagabbro were dated $3.46 \mathrm{Ga}$, the zircon from the charnockite, $2.66 \mathrm{Ga}$, the granulite metamorphism, 2.2 and $1.8 \mathrm{Ga}$ (zircon) and $1.95 \mathrm{Ga}$ (Sm-Nd mineral isochron) (a review in [Rosen et al., 1994]). Later, the orthopyroxene plagiogneisses were dated 3.1-3.2 Ga repeatedly using the upper discordia and concordia intersection for zircon. The superimposed process (the lower intersection of the same discordia) was dated 1.94-1.98 Ga [Rezvanova et al., 1994; Shemyakin et al., 1998]. The model age of the protolith was found to be $3.0 \mathrm{Ga}$ [Kotov et al., 1999]. A date of $2.9 \mathrm{Ga}$ based on a Rb-Sr isochrone [Mishkin et al., 1999] may date the local metamorphism associated with an ancient collision event. The poorly metamorphosed basalts of the superimposed Kholodnikan greenstone belt had been emplaced 2.15 Ga ago (Sm-Nd mineral isochron, [Rosen et al., 1994]).

Completing the discussion of the craton's structure, it should be noted that the collision-type granitoids and charnockites were the products of the final amalgamation. 


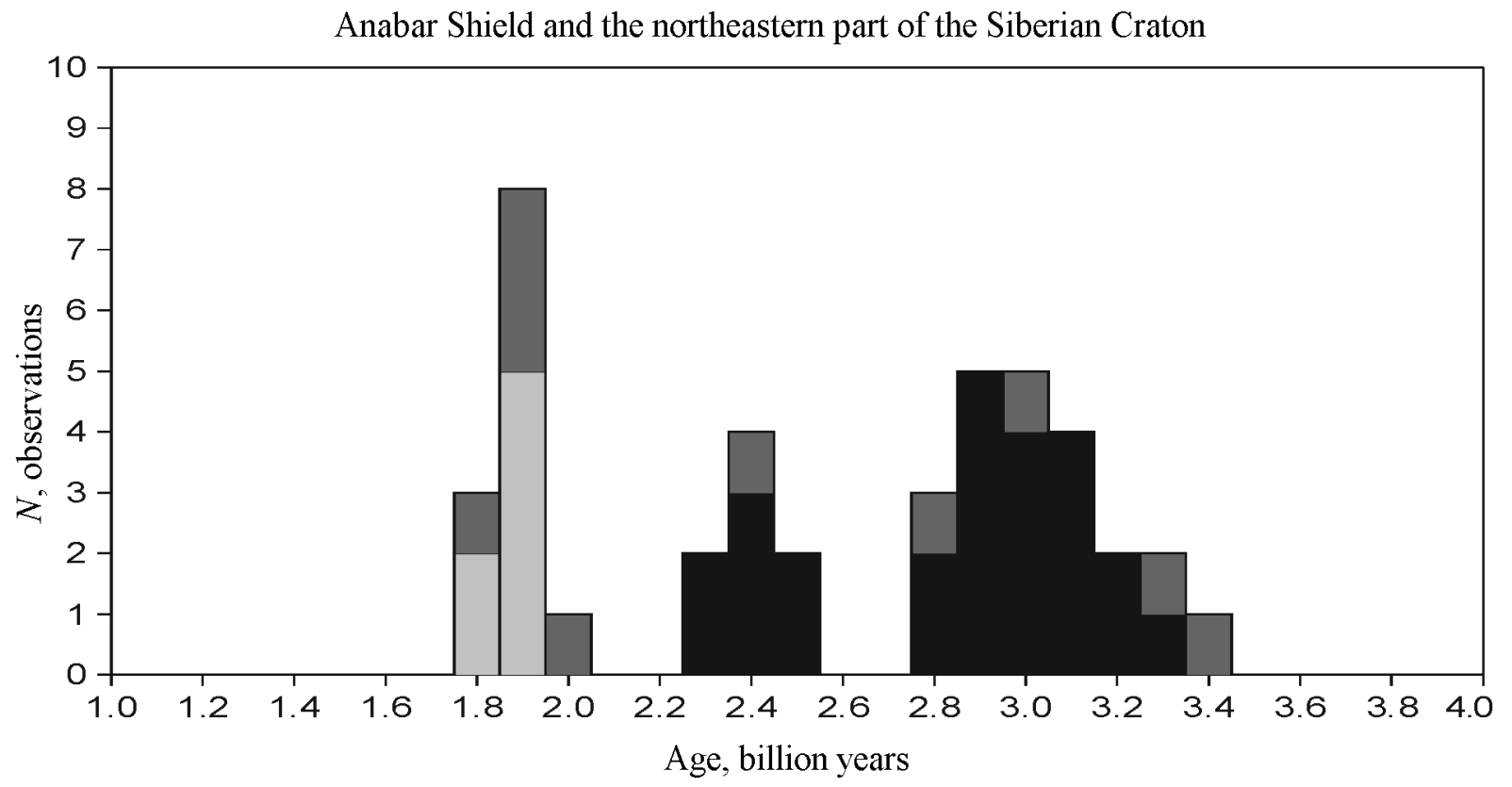

$$
\begin{aligned}
& \text { Sm-Nd model age, T(DM)Nd, }(N=23) \\
& \square \quad \text { Sm-Nd mineral isochron, }(N=7) \\
& \square-P b \text {, zircon, upper intersection, }(N=10)
\end{aligned}
$$

Figure 3. Histogram of isotope-geochronological dates for the Anabar Shield and the northeastern part of the craton, see the text for explanations.

It appears that a huge collision-type mountain building of the Himalayan type seems to have been produced $1.8 \mathrm{Ga}$ ago. The granitoids, melted during the collision, seem to have concentrated in the top of this structure [Rosen and Fedorovskii, 2001]. Propped by the crust of a great thickness, this mountainous region was eroded during the subsequent 150 million years, when the upper granite layer of the crust was locally eroded completely, the phenomenon obvious at least in the Anabar Shield. The Riphean gently dipping platform cover began to accumulate on the resulting peneplain $1.65 \mathrm{Ga}$ ago.

\section{Generalized Chronology of the Events}

The comparison of the isotopic datings in terms of their frequency allows one to reconstruct the general patterns of the craton's history. Our geochronologic isotope database includes 224 most reliable and geologically consistent datings. As far as the Siberian Craton is concerned, I used 144 datings including 88 datings reported in the literature before 1992 [Rosen et al., 1994], 56 datings after 1992 [Kovach, 1994; Kovach et al., 1999] (the $\mathrm{T}_{N d}(\mathrm{DM})$ datings for metamorphic graywackes, contradicting the isotopic data available, were discarded), and also the data reported by [Frost et al., 1998; Jahn et al., 1998; Kotov et al., 1999; Rezvanova et al., 1994; Shemyakin et al., 1998]. I also used 42 datings for the Anabar Shield and 36 datings for the Akitkan Belt ([Neimark et al., 1998] and other literature).

As far as the Aldan Shield and the Yenisey Highlands (without the Anabar Shield and the northeastern region), that is, the bulk of the Siberian Craton, are concerned, the model ages derived by the $\mathrm{Sm}-\mathrm{Nd}$ method, date the time when the depleted-mantle material was supplied to the granite-greenstone and granulite complexes as roughly 3.5 and 3.0 Ga ago (Figure 2). The $\mathrm{T}_{\mathrm{Nd}}(\mathrm{DM}) \mathrm{Nd}$ datings of $2.2-$ $2.4 \mathrm{Ga}$ date the events that occurred immediately after the formation of the microcontinents in association with the orogenesis (Ungra mafics, the greenstones of the Kholodnikan Belt, etc.). The dates ranging between 1.7 and $2.0 \mathrm{Ga}$ were obtained mainly from zircons and date the anatectic melting of silicic magma (migmatites, granitoids, and charnockites), which was caused by the heating of the crust thickened by collision.

The Anabar Shield and the northeastern part of the Siberian Craton were marked by the time-different and diverse processes of the formation of the terrane rocks and their collision-related transformation. In addition to the separation of migmatite from the depleted mantle 3.1-3.2 Ga ago, the datings available also date the migmatites and granitoids by zircons, and also the age of the granulite metamorphism coinciding with them in terms of Sm-Nd mineral isochrons $(1.8-2.0 \mathrm{Ga})$, as is obvious from Figure 3. The 


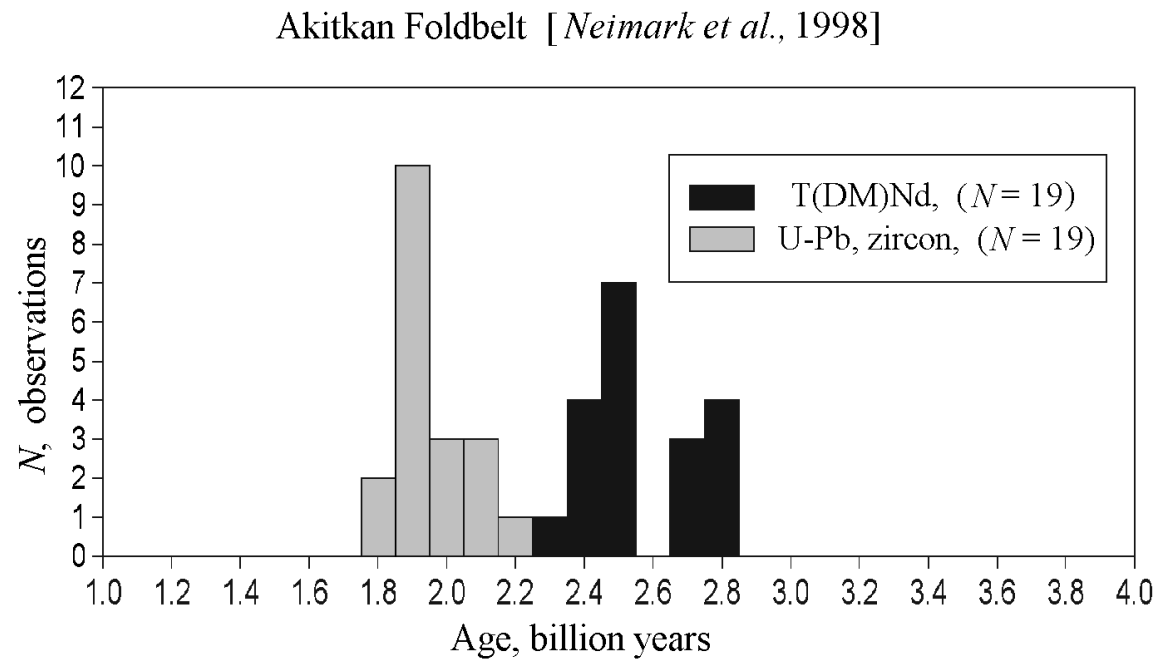

Figure 4. Histogram of isotope-geochronological dates for the Akitkan Foldbelt, see the text for explanations.

coincidence of this kind is the necessary condition for heating the thickened crust in a collision prism [England and Thompson, 1984]. A specific situation was discovered in the Akitkan Belt (Figure 4). Here, the U-Pb dates obtained by a U-Pb methods using zircons, ranging from 1.8 to $2.0 \mathrm{Ga}$, belong to the extrusive rocks with an Sm-Nd model age of $2.4 \mathrm{Ga}$ for the subduction source, whereas the sources of the contamination of the extrusive rocks with a model $\mathrm{Sm}-\mathrm{Nd}$ age of 2.5-2.8 Ga belong to a plate, subducted as a result of the collision, which in the present-day structure is included into the Anabar tectonic province.

The features, common for all histograms presented in Figures 2, 3, and 4, are the absence of model Sm-Nd datings younger than $2.2 \mathrm{Ga}$ and the predominance of datings obtained for zircons from migmatites and granites in the range of 1.8-2.0 Ga. It appears that during the accretion of the craton there was no addition of new material from the mantle, and the collision-related granitoids originated from the sialic material of the old microcontinents. Therefore the Siberian Craton was formed as a result of the accretion of Archean microcontinents which were transformed to tectonic blocks - granulite-gneiss and granite-greenstone terranes - connected along the collision zones. The juvenile material of the granulite-gneiss terranes had separated from the depleted mantle 3.3 and $3.0 \mathrm{Ga}$ ago, and that of the granite-greenstone terranes, 3.5 and $2.5 \mathrm{Ga}$ ago. The volcanic and sedimentary rocks of the foldbelts accumulated on their basements 2.4 and $2.1 \mathrm{Ga}$ ago. Collision (fault) zones developed in two periods of time, approximately 1.9 and $1.8 \mathrm{Ga}$ ago. The local metamorphism and granite formation in the collision zones were accompanied during each of these periods of time by synchronous areal granulite metamorphism in the adjacent terranes. This suggests a fairly homogeneous field of high pressures and temperatures, characteristic of the intermediate depths of the collision-thickened continental crust [England and Thompson, 1984; Rosen and Fedorovskii, 2001].

\section{Siberian, North American, and Other Cratons}

The above evidence suggests that the Siberian Craton is a fairly representative object for characterizing the process of the amalgamation of ancient sialic masses. In this work I used petrological criteria for recognizing the amalgamation of sialic blocks with the continents, namely granitoid formation, areal metamorphism in the collision prism, etc. The no less effective method for proving the amalgamation processes is the analysis of the processes of tectonic stabilization, the accumulation and deformation of sedimentary covers, and other processes [Khain, 2001]. This method was not used in this study because of the specific features of the Siberian Craton and, primarily, because of its larger part being covered by Riphean-Phanerozoic rocks. For this reason its comparison with other cratons, offered below, is based on the petrologic and geochronologic analyses.

The reconstructions reported by Vernikovskii [1996], Condie and Rosen [1994], Khudoley et al. [2001], and others researchers proved that by the end of the Paleoproterozoic the Siberian Craton constituted a united whole with the North American Craton. It appears that the North American Craton (Laurentia) and the Siberian Craton existed as one megacraton by the end of the collision (orogenic) epoch 1.8 Ga ago (Figure 5) [Condie and Rosen, 1994]. This is proved by the agreement of the datings and geological positions of the oldest rocks from the Slave Province of the North American Craton and from the opposite Aldan Province of the Siberian Craton. Also coincident are the positions of the foldbelts superimposed over this basement and deformed 2.0-1.9 Ga ago, which outcrop both in the Victoria Island of the north American Craton, and in the Aekite and Khapchan foldbelts of the Siberian Craton. This coincidence is most evident from the comparison of the Akitkan Foldbelt, composed of island-arc volcanics, thrust 


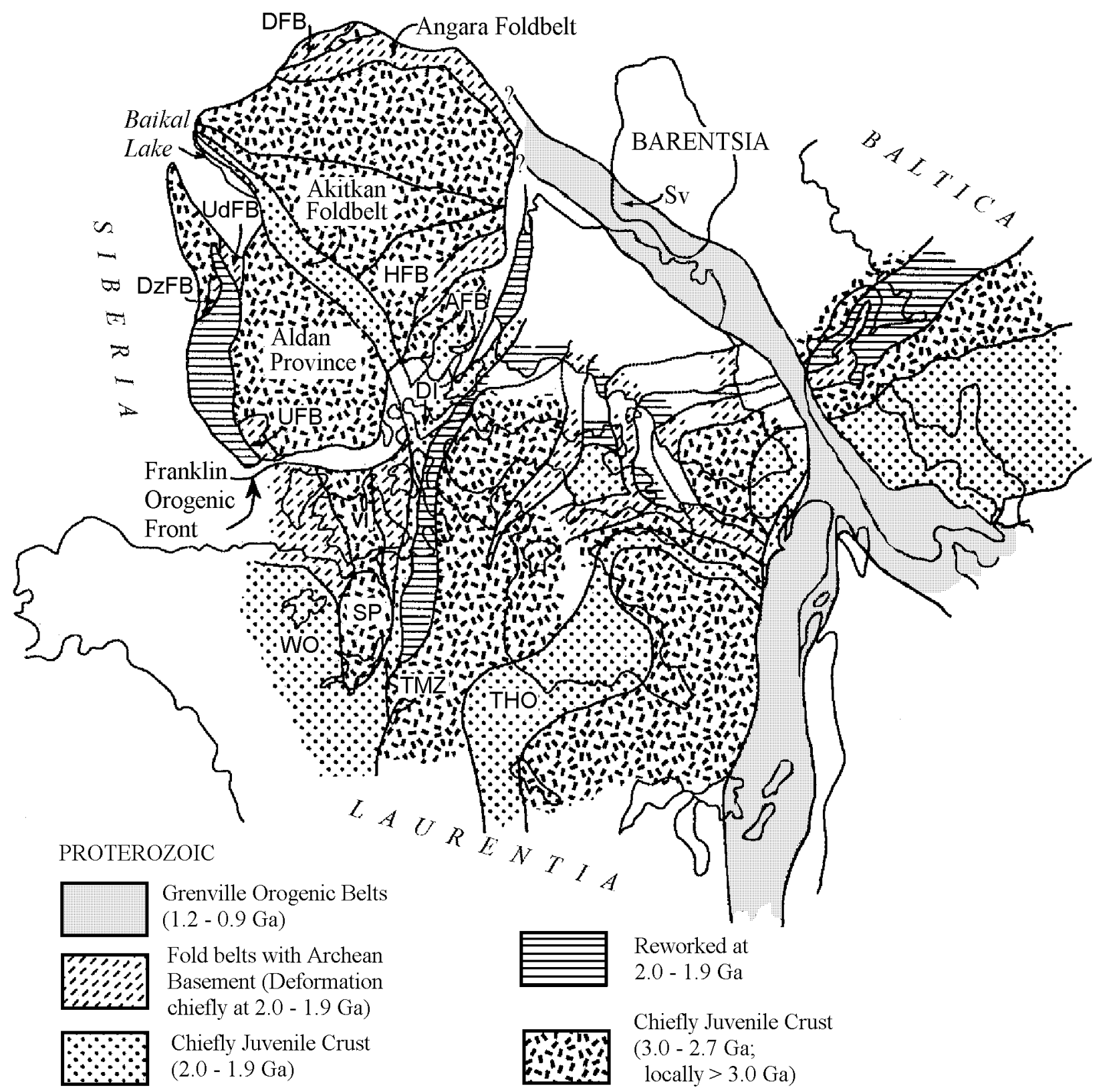

Figure 5. Reconstruction of the Proterozoic Supercontinent for 2.0-1.9 and 0.8 Ga ago. Abbreviated names of foldbelts: AFB - Aekite Foldbelt, DzFB - Dzheltulak Foldbelt, UdFB - Udokan Foldbelt, UFB - Ulkan Foldbelt, KhFB - Khapchan Foldbelt; other structural units: TMZ - Telon magmatic zone, WO - Wopmai Orogen, Sv - Svalbard; DI - Devon Island; VI - Victoria Island; BI - Baffin Land; SP - Slave Province, THO - Trans-Hudson Orogen.

over the active margin of the Anabar Paleocontinent, with the Telon igneous-rock arc which includes both island-arc volcanics and igneous rocks that accumulated on the active continental margin (Figure 5).

In fact, a similar chronological sequence of the collisionrelated igneous rocks has been found in the Canadian Shield, where the marginal basins of the Superior Province (where the crust originated $3.22-2.74 \mathrm{Ga}$ ago) were formed $2.04-$ $1.92 \mathrm{Ga}$ ago, the collision-related overthrusting took place
1.79-1.82 Ga ago with the subducted plate travelling a distance as great as $160 \mathrm{~km}$ (Trans-Hudson orogenic belt, [StOnge et al., 1999], Figure 5). Overthrusting manifested itself also in granulite metamorphism and synchronous granite formation [Jackson and Berman, 2000; St-Onge et al., 2000]. This movement was followed immediately by post-collision isostatic-compensation rising $(1.8 \mathrm{Ga}$, Ray Province [Scott, 1998]). The subduction- and collision-related granites were intruded 1.96-1.99 and 1.93-1.95 years ago, respectively, also 
on the western margin of the Churchill Province, in the Telon volcanic arc [Jackson and Berman, 2000; McDonough et al., 1995] (Figure 5). These data confirm the existence of the Siberia-Laurentia Paleocontinent [Condie and Rosen, 1994]. Its separation into the Siberia and Laurentia continents seems to have occurred in the late Riphean $0.8-0.7 \mathrm{Ga}$ ago [Yarmolyuk and Kovalenko, 2001].

The other cratons, now isolated in different continents, also show a similar sequence of geologic events. For instance, in the Limpopo Belt of the African Continent, magmatic activity occurred $3.2 \mathrm{Ga}$ ago, granulite metamorphism and charnockite magmatism took place 2.6-2.7 Ga ago, a collision between the Zimbabwe and Kaapval cratons, crustal thickening, and metamorphism occurred 2.05-1.95 Ga ago [Holzer et al., 1998], these activities being accompanied by the formation of granites [Kröner et al., 1999] and charnockites [Schaller et al., 1999]. The last accretion event [Kouamelen et al., 1997] and the latest granite formation [Ring et al., 1997] were dated ca. 2.0 Ga ago, these processes being widely recorded in the other regions of Africa. In the Baltic Shield, post-collision granites were formed by melting $1.9 \mathrm{Ga}$ ago [Elliot et al., 1998]. Crustal granites were produced by the melting of the Archean basement during the accretion of the craton $1.8 \mathrm{Ga}$ ago [Öhlander et al., 1999], simultaneously with the emplacement of a shoshonite series [Eklund et al., 1998].

In Australia, the post-collision high-K granites, shallow intrusions, and volcanic rocks were emplaced $1.85-1.865 \mathrm{Ga}$ ago as a result of the partial melting of the calc-alkalic and medium to acid crustal rocks during the accretion of the early Paleoproterozoic terranes to the southern and Eastern boundaries of the Kimberley Craton [Griffin et al., 2000]. In China, approximately $1.8 \mathrm{Ga}$ ago, high-pressure metamorphism and the subsequent rapid exhumation took place in association with the amalgamation of the Eastern and Western blocks, which resulted in the origin of the North China Craton [Zhao et al., 2001]. The amalgamation of the South American Craton from different terranes took place during the Paleoproterozoic, 2.1 Ga ago [Alkmim and Marshak, 1998]. It follows from this short review of collision-related magmatism that the processes of ancient terrane amalgamation occurred during the Late Paleoproterozoic in all modern continents.

\section{Paleoproterozoic Supercontinent}

The chronology of the collision events based on their main petrologic markers, namely, collision-related granitoids, shows that collision-related granite formation took place everywhere at the time between 1.8 and $1.9 \mathrm{Ga}$. This proves the existence of a huge collision region (supercontinent), although direct (paleomagnetic) data for compiling a tectonic map for this structure are still insufficient. It seems unlikely that the entire continental crust of the Earth was combined into one supercontinent more than one time in its geologic history.

The apparent chronological periodicity was obviously associated with the manifestation of the global cycles of the ac- cretion and break-up of the supercontinents from one Pangea to another [Dobretsov and Kirdyashkin, 1994]. It is believed that Pangea- 0 arose about $2.5 \mathrm{Ga}$ ago at the boundary between the Archean and Proterozoic [Khain, 2000, 2001]. Pangea-1 [Khain, 2001] or Paleopangea [Piper, 2000] is believed to have originated at the end of the Paleoproterozoic, 2.0-1.7 Ga ago. The Siberian-Laurentian part of that Pangea has been discussed above. The age range of the later supercontinents was investigated by Khain [2001], Condie [1998], and many other geoscientists. The causes of the origin and break-up of the supercontinents are discussed actively in literature. The summary that follows is based on the ideas proposed by Condie [1998], which are distinguished by their simplicity and clearness.

The mechanisms responsible for this periodicity were attributed supposedly to changes in the conditions of the mantle convection. The whole-mantle convection produced one convection cell, the effect of which resulted in the sialic mass accumulation at some point in the descending flow and in the origin of a supercontinent [Condie, 1998]. This system arose after the avalanche of the material of the subducted oceanic crust accumulated at the base of the upper mantle into the lower mantle. The more intense heating under the crust of the supercontinent violated the equilibrium in a one-cell convection system. A multicell convection arose separately in the lower and in the upper mantle. Eventually, this resulted in the breakup of the supercontinent and in the operation of the mechanism of individual lithospheric plate tectonics [Condie, 1998].

In the geologic history of the Earth, the first period of the active addition of the juvenile material into the continental crust was the time interval of 3.0-2.7 Ga under the conditions of a one-layer and one-cell mantle convection. This activity was terminated by the accretion of continental masses and the formation of a late Archeozoic Supercontinent [Condie, 1998]. It was apparently during that period of time that the bulk of the juvenile material was injected into the crust of the Siberian Craton (59\% of the total number of Sm-Nd model datings covering the age range of $3.3-2.7 \mathrm{Ga}$ [Rosen et al., 2000] (Figures 2-4)). It appears that the granulite metamorphism, dated 2.5-2.8 Ga and proved in several terranes, marks the time of the collision termination and of the origin of the first supercontinent. Nothing is known thus far concerning its geological configuration.

At the beginning of the second period, under the conditions of a multicell mantle convection, the first supercontinent broke into individual fragments, and a new portion of juvenile material was added [Condie, 1998]. This breakup seems to have produced the microcontinents which were connected later to form the Siberian Craton. During the repeated switching to a one-layer convection sialic masses were accreted to produce another, Paleoproterozoic, supercontinent with an age of 2.1-1.7 Ga [Condie, 1998]. It was this stage that was most widely manifested in the Siberian Craton in the form of collision processes. A unique feature of this craton is that by that time a negligibly small amount of juvenile material was produced: merely a few percent of the total amount of magmatic rocks, the model Sm-Nd ages ranging over the interval of 2.01-1.8 Ga [Rosen et al., 2000] (Figures 2-4). Consequently, granites were produced 
by the partial melting of some ancient crustal rocks, and the resulting granite melt flowed later upward from the lower crust where a contaminated granulite restite was produced simultaneously in a complimentary manner [Rosen and $\mathrm{Fe}$ dorovskii, 2001].

By and large, it can be stated that the Siberian Paleoproterozoic Supercontinent was produced from the fragments of the first Late Archean Supercontinent, these fragments being microcontinents prior to their amalgamation. Paleoproterozoic sedimentary covers and/or volcanic rocks, accompanied by comagmatic intrusions, accumulated in some regions. During the collision and amalgamation of the microcontinents, acid crustal magmas rose into the upper crust of the collision systems. No mantle material was added, this being a distinctive feature of the Siberian fragment of the Paleoproterozoic Supercontinent.

\section{Conclusion}

The sialic (volcanogenic) matter of the terranes in the Siberian fragment of the Paleoproterozoic Supercontinent separated 3.5, 3.3, 3.0, and 2.5 Ga ago. The collision sutures between the terranes are dated by the granitoids that melted from the sialic crust of the terranes in two periods of time, approximately 1.9 and $1.8 \mathrm{Ga}$ ago. The local metamorphism and granite formation inside the collision (fault) zones were accompanied by synchronous areal dry granulite metamorphism in the adjacent terranes. This suggests the fairly high, uniform temperatures and pressures of a thicker crust in the collision prism. The amalgamation of the continental crust fragments was completed by the formation of a huge collision-type mountain building about $1.8 \mathrm{Ga}$ ago, which was totally eroded later. Early Riphean platformtype clastic deposits began to accumulate on the resulting peneplain $1.65 \mathrm{Ga}$ ago, that is, 150 million years after the completion of the collision processes.

The Siberian Craton is a part of the Paleoproterozoic Supercontinent (Pangea-1) produced by the fragments of a dismembered Archeozoic supercontinent (Pangea-0), all fragments being microcontinents with their own Paleoproterozoic sedimentary-volcanogenic covers, transformed after collision to foldbelts, superimposed over the basements of the ancient terranes. The exception was the Paleoproterozoic Akitkan volcanic belt, which evolved as an island arc and later, in the course of the total amalgamation, was thrust over the ancient basement and involved in anorogenic silicic magmatism (A-granites).

Acknowledgments. I am very grateful to V. A. Vernikovskii, A. P. Smelov, V. S. Fedorovskii, and V. E. Khain for the useful discussion of various aspects of the problems involved. This study was supported by the Russian Foundation for Basic Research, Project 00-05-64784.

\section{References}

Aftalion, M., E. V. Bibikova, D. R Bowes, A. M. Hopgood, and L. L. Perchuk, Timing of Early Proterozoic collisional and extensional events in the granulite-gneiss-granite complex, Lake Baikal, USSR: A U-Pb, RB-Sr, and SM-Nd isotopic study, J. Geol., 99, 851-862, 1991.

Alkmim, F. F., and S. Marshak, Transamazonian orogeny in the southern Sao Francisco Craton region, Minas Gerais, Brazil: Evidence for Paleoproterozoic collision and collapse in the Quadrilatero Ferrifero, Precamb. Res., 90, (1-2), 29-58, 1998.

Bibikova, E. V., G. M. Drugova, V. L. Duk, O. A. Levchenkov, and I. M. Morozova, Geochronology of the Vitim-Aldan Shield, in Methods of Isotope Geochronology and Geochronological Scale, pp. 135-189, Nauka Press, Moscow, 1986.

Chernykh, A. I, Geological Structure, Petrology and Geochemistry of the Precambrian Ophyolites and Paleoisland-Arc Rocks from the Enisei Highlands, Abstract of Candidate Geol.-Mineral Sci. Dissertation, Novosibirsk, Institute of Geology, Siberian Division, Russian Academy, 2000.

Condie, K. C., Episodic continental growth and supercontinents: A mantle avalanche connection?, Earth Planet. Sci. Lett., 163, 97-108, 1998 .

Condie, K. C., and O. M. Rosen, Laurentia-Siberia connection revisited, Geology, 22, 168-170, 1994.

Dobretsov, N. L., Ed., The Early Precambrian of Southern Yakutia, Nauka Press, Moscow, 1986.

Dobretsov, N. L., and A. G. Kirdyshkin, Deep-Earth Geodynamics, OIGGM RAN Press, Novosibirsk, 1994.

Eklund, O., D. Konopelko, H. Rutanen, S. Fröjdö, S., and A. D. Shebanov, $1.8 \mathrm{Ga}$ Svecofennian post-collisional shoshonitic magmatism in the Fennoscandian Shield, Lithos 45, (1-4), 87-108, 1998.

Elliot, B. A., Tarani Rämö, and M. Nironen, Mineral chemistry constraints on the evolution of the $1.88-1.87$ Ga postkinematic granite plutons in the central Finland granitoid complex, Lithos, 45, (1-4), 109-129, 1998.

England, P. C., and B. Thompson, Pressure-temperature-time paths of regional metamorphism, J. Petrol. 25, (4), 894-955, 1984.

Frost, B. R., O. V. Avchenko, K. R. Chamberlain, and C. D. Frost, Evidence for extensive Proterozoic remobilization of the Aldan Shield and Implications for the Proterozoic plate tectonic reconstructions of Siberia and Laurentia, Precamb. Res., 89, (1-2), 1-23, 1998.

Godzevich, B. L., Archean stratigraphy of the Southern AldanStanovoi Shield, in Problems of the Early Precambrian Stratigraphy in Central Siberia, pp. 127-136, Nauka Press, Moscow, 1986.

Griffin, T. J., R. W. Page, S. Sheppard, and I. M. Tyler, Tectonic implications of Paleoproterozoic post-collisional, high-K felsic igneous rocks from the Kimberley region of northwestern Australia, Precamb. Res., 101, 1-23, 2000.

Holzer, L., R. Frei, and J. M. Barton, Jr., Unraveling the record of successive high-grade events in the Central Zone of the Limpopo Belt using $\mathrm{Pb}$ single-phase dating of metamorphic minerals, Precamb. Res., 87, (1-2), 87-115, 1998.

Jackson, G. D., and R. G. Berman, Precambrian metamorphic and tectonic evolution of northern Baffin Island, Nunavut, Canada, Can. Mineral., 38, 399-421, 2000.

Jahn, B.-M., and H. Cuvellier, $\mathrm{Pb}-\mathrm{Pb}$ and $\mathrm{U}-\mathrm{Pb}$ geochronology of carbonate rocks: An assessment, Chem. Geol., 115, 125-151, 1998.

Jahn, B.-M., G. Gruau, R. Capdevila, et al., Archean crustal evolution of the Aldan Shield, Siberia: Geochemical and isotopic constraints, Precamb. Res., 91, 333-363, 1998.

Khain, V. E., Problems of Early Precambrian tectonics, Vestn. Mosk. Univ., (4), 13-23, 2000.

Khain, V. E., Tectonics of the Continents and Oceans, Nauchnyi Mir, Moscow, 2001.

Khudoley, A. K., R. H. Rainbird, et. al., Sedimentary evolution of the Riphean-Vendian basin in southeastern Siberia, Precamb. Res., 111, 129-163, 2001. 
Kotov, A. B., I. M. Shemyakin, E. B. Salnikova, and V. P. Kovach, The formation history and isotopic structure of the continental crust in the Sutam block of the Aldan Shield, Doklady Akad. Nauk, 366, (6), 809-812, 1999.

Kouamelen, A. N., C. Delor, and J. J. Peaucat, Geochronological evidence for the reworking of Archean terranes during the Early Proterozoic $(2.1 \mathrm{Ga}$ ) in the western Cote d'Ivoire (Man Rise West African craton), Precamb. Res., 86, (3-4), 177-199, 1997.

Kovach, V. P., A. B. Kotov, V. I. Berezkin, et al., Age thresholds in the formation of supracrustal highly metamorphosed rocks in the central part of the Aldan Shield: Sm-Nd isotope data, Stratigrafiya, Geol. Korrelyatsiya, 7, (1), 3-17, 1999.

Kovach, V. P., Granitoid Rocks of the Central Aldan GranuliteGneiss Province: Geology and Petrology, Abstract of Candidate Sci. Dissertation, IGGD RAN Press, St. Petersburg, 1994.

Kröner, A., P. Jaeckel, G. Brandl, et al., Single zircon ages for granitoid gneisses in the Central Zone of the Limpopo Belt, South Africa: Geodynamic significance, Precamb. Res., 93, (4), 299-337, 1999.

Larin, A. M., Yu. V. Amelin, L. A. Neymark, and R. Sh. Krimsky, The origin of the 1.73-1.70 Ga anorogenic Ulkan volcanoplutonic complex, Siberian Craton, Russia: Inferences from geochronological, geochemical and $\mathrm{Nd}-\mathrm{Sr}-\mathrm{Pb}$ isotopic data, Ann. Acad. Bras. Ci., 69, (3), 295-312, 1997.

Luts, B. G., and V. K. Oksman, Deeply Eroded Fault Zones in the Anabar Shield, Nauka Press, Moscow, 1990.

Markov, M. S., Ed., The Archean of the Anabar Shield and the Problems of the Early Evolution of the Earth, Nauka Press, Moscow, 1988.

McDonough, V. R., V. J. McNicoll, and E. M. Schetselar, Age and kinematics of crustal shortening and escape in a two-sided oblique-slip collisional and magmatic orogen, Paleoproterozoic Taltson magmatic zone, northeastern Alberta, in Ross, G. M., ed., Alberta Basement Transects Workshop, LITHOPROBE Report 47, pp. 264-309, University of British Columbia, 1995.

Mekhonoshin, A. S., Basic-ultrabasic magmatism of the Onot Greenstone Belt (Eastern Sayan), Geologiya $i$ Geofizika, 40, (12), 1772-1784, 1999.

Mishkin, M. A., M. N. Maslovskaya, S. N. Lavrin, and G. M. Vovna, Geochemistry and Rb-Sr isotopy of metabasites and enderbites from the south of the Aldan Shield (Sutam Block), Geokhimiya, (9), 931-940, 1999.

Neimark, L. A., A. M. Lavrin, A. A. Nemchin, et al., Geochemical, geochronological (U-Pb) and isotopic ( $\mathrm{Pb}, \mathrm{Nd}$ ) evidence of anorogenic magmatism in the North Baikal volcanoplutonic belt, Petrologiya, 6, (2), 139-164, 1998.

Nozhkin, A. D., The Early Proterozoic continental-margin rocks of the Angara foldbelt and their metallogeny, Geologiya $i$ Geofizika, 40, (11), 1524-1544, 1999.

Öhlander, B., C. Mellquist, and T. Skiöld, Sm-Nd isotope evidence of a collisional event in the Precambrian of Northern Sweden, Precamb. Res., 93, (1), 105-117, 1999.

Petrov, A. F., G. S. Gusev, F. F. Tretyakov, and V. S. Oksman, Archean (Aldan) and Early Proterozoic (Karelia) megacomplexes, in V. V. Kovalskii, Ed., Structure and Evolution of the Earth's Crust in Yakutia, pp. 9-39, Nauka, Moscow, 1985.

Piper, J. D. A., The Neoproterozoic supercontinent: Rodinia or Paleopangea?, Earth Planet. Sci. Lett., 176, 131-146, 2000.

Popov, N. V., and A. P. Smelov, The metamorphic rocks of the Aldan Shield, Geol. Geofiz. 37, (1), 148-161, 1996.

Rezvanova N. G., O. A. Levchenkov, I. S. Bogomolov, et al., Comparison of zircon-phase separation techniques for geochronological studies, Geokhimiya, (7), 1076-1086, 1994.

Ring, U., A. Kröner, and P. Touelkeridis, Paleoproterozoic granulite-facies metamorphism and granitoid intrusions in the Ubendian-Usadaran Orogen of northern Malavi, eastern-central Africa, Precamb. Res., 85, (1-2), 27-51, 1997.

Rosen, O. M., and V. S. Fedorovskii, Collision Granitoids and Crustal Layering, Nauchnyi Mir, Moscow, 2001.

Rosen, O. M., A. D. Nozhkin, and K. C. Condie, Active margin of the Tungus Protocontinent in the Early Proterozoic: The earliest shoreline of the Paleo-Asian Ocean?, in: N. L. Dobretsov and N. A. Berzin, eds., 4th Int. Symp. on Dynamic Evolution of Paleoasian Ocean, p. 117, UIGGM, Novosibirsk, 1993.

Rosen, O. M., K. C. Condie, L. M. Natapov, and A. D. Nozhkin, Archean and early Proterozoic evolution of the Siberain Craton: A preliminary assessment, in: K. S. Condie, ed., Archean Crustal Evolution, pp. 411-459, Elsevier, Amsterdam, 1994.

Rosen, O. M., D. Z. Zhuravlev, M. K. Sukhanov, et al., Differences in the isotope geochemistry and age of Early Proterozoic terranes, collision zones, and associated northosites in the northeast of the Siberian Craton, Geol. Geophys., 41, (2), 163-179, 2000.

Rundkvist, D. V., and F. P. Mitrofanov, Eds., Precambrian Geology of the USSR Territory, Nauka, Leningrad, 1988.

Schaller, M., O. Steinger, I. Studer, et al., Exhumation of Limpopo central-zone granulites and dextral transcurrent movement at 2.0 Ga along the Palara shear zone, Northern Province, South Africa, Precamb. Res. 96, 263-288, 1999.

Scott, D. J., An overview of the U-Pb geochronology of the Paleoproterozoic Torngat Orogen, Northwestern Canada, Precamb. Res. 91, (1-2), 91-107, 1998.

Sharkov, E. V., M. M. Bogina, J. E. Quick, and A. S. Mekhanoshin, Tectonic blocks of the Precambrian lower crust and upper mantle, Southern Sayan Mountains, Eastern Siberia, Intern. Geol. Rev., 37, 81-91, 1995.

Shemyakin, V. M., V. A. Glebovitskii, N. G. Berezhnaya, et al., The age of the oldest rocks in the Sutam crustal block, Dokl. Russian Academy, 360, (4), 526-529, 1998.

Sklyarov, E. V., D. P. Gladkochub, A. M. Mazukabzov, and $\mathrm{Yu}$. A. Menshagin, Metamorphism of ancient ophyolites in the Sharyzhalgai Uplift, Geologiya i Geofizika, 39, (12), 1733-1749, 1998.

Smelov, A. P., A. N. Zedgenizov, L. M. Parfenov, and V. F. Timofeev, Precambrian terranes in the Aldan-Stanovoi Shield, in Metallogeny, Oil Prospects and Geodynamics of the North Asian Craton and Surrounding Foldbelts, pp. 119-120, Irkutsk, Siberian Division of the Russian Academy, Santai Press, 1998.

Smelov, A. P., and V. I. Beryozkin, Retrograded eclogites in the Olekma granite-greenstone region, Aldan Shield, Siberia, Precamb. Res. 62, 419-430, 1993.

St-Onge, M. R., S. B. Lucas, D. J. Scott, and N. Wodicka, Upper and lower plate juxtaposition, deformation, and metamorphism during crustal convergence, Trans-Hudson orogen (Quebec-Baffin segment), Canada, Precamb. Res., 93, (1), 2749, 1999.

St-Onge, M. R., N. Wodicka, and S. B. Lucas, Granulite- and amphibolite-facies metamorphism in a convergent-plate margin setting: Synthesis of the Quebec-Baffin segment of the TransHudson Orogen, Can. Mineral., 38, 379-398, 2000.

Vernikovskii, V. A., Geodynamic Evolution of the Taimyr Fold Region, CO RAN Press, Novosibirsk, 1996.

Vernikovskii, V. A., A. E. Vernikovskaya, E. B. Dalnikova, et al., New U-Pb data timing the formation of the Predivino Terrane of the Enisei Highlands, Geologiya i Geofizika 40, (2), 255-259, 1999.

Yanshin, F. L., and C. B. Borukaev, The Earth's crust of Siberia: Tectonics and Evolution, Transactions of IGiG SO RAN, iss. 173, Nauka, Novosibirsk, 1988.

Yarmolyuk, V. V., and V. I. Kovalenko, Late Riphean intraplate magmatism in Southern Siberia as a trace of splitting from Laurentia, in Supercontinents in Precambrian Geologic History, Irkutsk, Institute of the Earth's Crust, pp. 325-328, Siberian Division, Russian Academy of Sciences, 2001.

Zhao, G., A. Wilde, P. A. Cawood, and M. Sun, Archean blocks and their boundaries in the North China Craton: Lithological, geochemical, structural, and $\mathrm{P}-\mathrm{T}$ path constraints and tectonic evolution, Precamb. Res. 107, (1-2), 45-73, 2001.

Zonenshain, L. P., V. I. Kuzmin, and L. M. Natapov, Geology of the USSR: A plate tectonic synthesis, Amer. Geophys. Union Geodynamic Series 21, 242 p., 1989.

(Received 18 April 2002) 